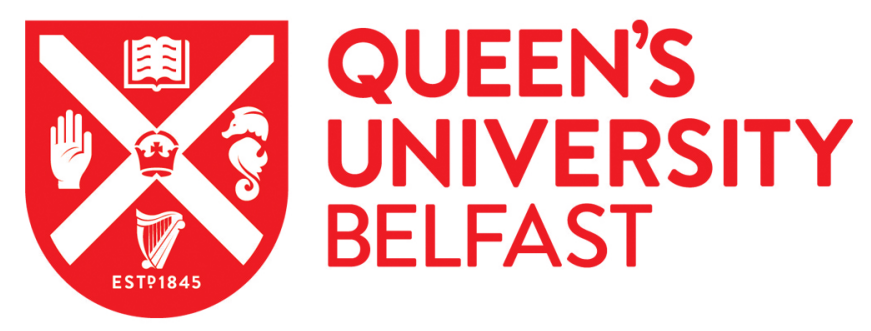

\title{
Generalized Wave Digital Filter Realizations of Arbitrary Reciprocal Connection Networks
}

Bernardini, A., Werner, K. J., Smith, J., \& Sarti, A. (2018). Generalized Wave Digital Filter Realizations of Arbitrary Reciprocal Connection Networks. IEEE Transactions on Circuits and Systems I: Regular Papers, 66(2), 694-707. https://doi.org/10.1109/TCSI.2018.2867508

Published in:

IEEE Transactions on Circuits and Systems I: Regular Papers

Document Version:

Peer reviewed version

Queen's University Belfast - Research Portal:

Link to publication record in Queen's University Belfast Research Portal

Publisher rights

(c) 2018 IEEE. This work is made available online in accordance with the publisher's policies. Please refer to any applicable terms of use of the publisher.

\section{General rights}

Copyright for the publications made accessible via the Queen's University Belfast Research Portal is retained by the author(s) and / or other copyright owners and it is a condition of accessing these publications that users recognise and abide by the legal requirements associated with these rights.

Take down policy

The Research Portal is Queen's institutional repository that provides access to Queen's research output. Every effort has been made to ensure that content in the Research Portal does not infringe any person's rights, or applicable UK laws. If you discover content in the Research Portal that you believe breaches copyright or violates any law, please contact openaccess@qub.ac.uk. 


\title{
Generalized Wave Digital Filter Realizations of Arbitrary Reciprocal Connection Networks
}

\author{
Alberto Bernardini, Student Member, IEEE, Kurt James Werner, Member, IEEE, \\ Julius O. Smith III, Member, IEEE, Augusto Sarti, Senior Member, IEEE
}

\begin{abstract}
In this paper, an existing approach for modeling and efficiently implementing arbitrary reciprocal connection networks using Wave Digital scattering junctions based on voltage waves is extended to be used in a broader class of Wave Digital Filters based on different kinds of waves. A generalized wave definition which includes traditional voltage waves, current waves and power-normalized waves as particular cases is employed. Closed-form formulas for computing the scattering matrices of the junctions are provided. Moreover, the approach is also extended to the family of Biparametric Wave Digital Filters, which have been recently introduced in the literature.
\end{abstract}

Index Terms-Wave Digital Filters, Scattering Matrices, Junctions, Adaptors, Connection Networks.

\section{INTRODUCTION}

$\mathbf{O}$ VER forty years ago, Martens and Meerkötter presented a method [1] for modeling arbitrary circuit topologies using multi-port Wave Digital (WD) adaptors [2]. The method was initially based on a partition of the digraph representing the reference circuit into tree and cotree. It was later extended to accommodate more general reciprocal connection networks, which embed reciprocal multi-ports, such as ideal transformers [3]-[6]. Recently, an alternative approach, based on the Modified Nodal Analysis (MNA) [7]-[11], was developed for implementing in the WD domain arbitrary connection networks, which also contain active and non-reciprocal linear multiports, such as controlled sources, nullors, and gyrators. The MNA-based method discussed in [7] allows us to implement a larger class of WD junctions than the approach presented in [1], [5], [6] for the two following reasons. The former is that the MNA-based method accommodates both reciprocal and non-reciprocal connection networks in the WD domain, while the applicability of the method in [1], [5], [6] is limited to reciprocal connection networks. The second reason is that the formulation of the method in [7] is based on a generalized definition of wave signals [12], which includes definitions of voltage, current and power-normalized waves of traditional WD Filters (WDFs) [13] as particular cases, while the results in [1], [5], [6] are limited to WDFs based on voltage waves. However, as outlined in [7], the method presented in [1], [5],

A. Bernardini and A. Sarti are with the Dipartimento di Elettronica, Informazione e Bioingegneria (DEIB); Politecnico di Milano, Piazza L. Da Vinci 32, 20133 Milano, Italy (e-mail: [alberto.bernardini,augusto.sarti] @ polimi.it). K. J. Werner is with the Sonic Arts Research Centre (SARC); School of Arts, English and Languages; Queen's University Belfast; 4 Cloreen Park, Belfast, BT9 5HN UK (e-mail: k.werner@qub.ac.uk). J. O. Smith III is with the Center for Computer Research in Music and Acoustics (CCRMA), Stanford University; 660 Lomita Drive, Stanford, California 94305-8180, USA (e-mail: jos@ccrma.stanford.edu).
[6] remains more efficient for realizing reciprocal connection networks in the WD domain. Therefore, it would be desirable to develop extensions of the method in [1], [5], [6] that are applicable to WD structures based on other types of waves, in order to investigate whether they can bring advantages over the MNA-based method [7] in implementing reciprocal junctions.

Reciprocity in the WD domain is an important property, as it can be exploited for a variety of theoretical derivations. For example, in [14] and [15], reciprocity is used to prove a theorem granting the convergence of a computationally inexpensive WD relaxation algorithm, called Scattering Iterative Method (SIM), which is suitable for solving circuits with multiple nonlinear one-ports characterized by monotonic $i-$ $v$ characteristics. The application of SIM to the case of large photovoltaic arrays of arbitrary topologies is described in [14], [16]. This fact constitutes a further motivation to explore generalizations of the method presented in [1], [5], [6], since, for instance, closed-form expressions of scattering matrices describing WD junctions based on different types of wave definitions could be useful for deriving extensions of the theorem proven in [14].

Various definitions of wave signals with diverse benefits are available in the literature of WDFs [13]. Among these, power-normalized waves (as defined in microwave engineering [17]) are worth mentioning for their energy properties in fixed-point implementations and for the orthogonality of their scattering matrices [18], [19]. In the literature on sound synthesis through physical modeling, for example, powernormalized waves have been employed for implementing WD models of acoustic systems involving WDFs and/or digital waveguides [20]-[22]. The generalized definition of waves, including voltage, current and power-normalized waves of traditional WDFs as particular cases, presented in [7], [12], allows us to derive general expressions of scattering relations which are parametrized in terms of the wave type. Moreover, a new family of Biparametric WDFs (BWDFs) was introduced in [23]. Wave signals in BWDFs are defined as depending on two free parameters per port instead of one, which enables the design of WD structures with increased degrees of freedom. Additional free parameters in BWDFs allow, for instance, the design of scattering matrices of series or parallel adaptors that are made of off-diagonal entries equal to -1 or +1 , thus, independent from the circuit parameters, and with diagonal entries equal to zero, making ports of adaptors all reflectionfree [23]. As only adaptors describing series and parallel interconnections were discussed in [23], a theoretical study on the design of adaptors representing arbitrary connection 
networks in BWDFs is missing in the literature. We believe that all the potential of recently introduced BWDFs have not yet been expressed and further special properties might be discovered, especially as the modeling and/or optimization of nonlinear WD structures is concerned [8], [14], [15]. For this reason, investigations on alternative ways of exploiting the free parameters are in progress.

In this article, extensions of the theory of Martens et al. [1], [5], [6] to the general definition of waves introduced in [7], [12] and to biparametric wave definitions [23] are presented. We will see that both extensions are not immediate. But the case of BWDFs is particularly interesting as the corresponding scattering matrices are far less constrained in their structure than those used in WDFs (e.g., they are no longer self-inverse). This makes the generalization of the results of Martens et al. to this case anything but straightforward.

The article is organized as follows. Section II provides a description of general reciprocal connection network, starting from the reciprocity condition that relates its port currents and port voltages. Section III shows how an arbitrary reciprocal connection network can be implemented in the WD domain using a scattering matrix based on the definition of wave variables presented in [7]. Section [V] presents two derivations of WD scattering matrices, based on two different biparametric definitions of wave variables [23]. Section V] explains how the scattering matrices derived in Section III and Section IV can be employed for the design of arbitrary adaptors in traditional WDFs and BWDFs. In particular, bridged-T and twin-T adaptors are derived, along with adaptors usable for implementing the Brune section and the Darlington-D section in the WD domain. Moreover, WD implementations of multiwinding ideal transformers with series and parallel structures are presented. We show that ports of adaptors based on biparametric definitions of waves can all be made reflection-free (i.e., can all be adapted). Examples of application of such adaptors for the implementation of non-trivial circuits are included in order to show the effectiveness of the proposed approach. Section VI presents a final discussion on the computational cost of the proposed method. Finally, the article is concluded, proposing some possible future developments.

\section{Reciprocal Connection Networks}

Let us consider a linear $N$-port reciprocal connection network characterized by a vector of port voltages $\mathbf{v}=$ $\left[v_{1}, \ldots, v_{N}\right]^{T}$ and a vector of port currents $\mathbf{i}=\left[i_{1}, \ldots, i_{N}\right]^{T}$, with $N>1$. We assume that $\mathbf{v}_{\mathrm{t}}$ is a column vector of size $q \times 1$, with $1 \leq q<N$, containing all $q$ independent port voltages, and that $\mathbf{i}_{1}$ is a column vector of size $p \times 1$, with $p=N-q$, containing all $p$ independent port currents. As discussed in [24], [25], there always exists a pair of matrices $\mathbf{Q}$ and $\mathbf{B}$ of sizes $q \times N$ and $p \times N$, respectively, such that

$$
\mathbf{v}=\mathbf{Q}^{T} \mathbf{v}_{\mathbf{t}}, \quad \mathbf{i}=\mathbf{B}^{T} \mathbf{i}_{\mathbf{l}} .
$$

Without loss of generality, we also assume that port variables are ordered in such a way that we can write $\mathbf{v}_{\mathrm{t}}=$ $\left[v_{N-q+1}, \ldots, v_{N}\right]^{T}$ and $\mathbf{i}_{1}=\left[i_{1}, \ldots, i_{p}\right]^{T}$. It follows that matrices $\mathbf{Q}$ and $\mathbf{B}$ can be expressed as [5], [6]

$$
\mathbf{Q}=\left[\begin{array}{ll}
\mathbf{Q}_{\mathrm{q}} & \mathbf{I}
\end{array}\right] \quad, \quad \mathbf{B}=\left[\begin{array}{ll}
\mathbf{I} & \mathbf{B}_{\mathrm{p}}
\end{array}\right],
$$

where $\mathbf{Q}_{\mathrm{q}}$ and $\mathbf{B}_{\mathrm{p}}$ are matrices of sizes $q \times p$ and $p \times q$, respectively, and $\mathbf{I}$ indicates a properly sized identity matrix.

Let us now consider two arbitrary and distinct pairs of vectors of port signals

$$
\begin{aligned}
& \left\{\tilde{\mathbf{v}}=\left[\tilde{v}_{1}, \ldots, \tilde{v}_{N}\right]^{T}, \tilde{\mathbf{i}}=\left[\tilde{i}_{1}, \ldots, \tilde{i}_{N}\right]^{T}\right\}, \\
& \left\{\hat{\mathbf{v}}=\left[\hat{v}_{1}, \ldots, \hat{v}_{N}\right]^{T}, \hat{\mathbf{i}}=\left[\hat{i}_{1}, \ldots, \hat{i}_{N}\right]^{T}\right\} .
\end{aligned}
$$

As the network is reciprocal, the reciprocity condition [26]

$$
\tilde{\mathbf{v}}^{T} \hat{\mathbf{i}}=\hat{\mathbf{v}}^{T} \tilde{\mathbf{i}}
$$

is always satisfied. We define $\tilde{\mathbf{v}}_{\mathrm{t}}, \tilde{\mathbf{i}}_{1}, \hat{\mathbf{v}}_{\mathrm{t}}$ and $\hat{\mathbf{i}}_{1}$ in such a way that, according to $(1), \tilde{\mathbf{v}}=\mathbf{Q}^{T} \tilde{\mathbf{v}}_{\mathrm{t}}, \tilde{\mathbf{i}}=\mathbf{B}^{T} \tilde{\mathbf{i}}_{1}, \hat{\mathbf{v}}=\mathbf{Q}^{T} \hat{\mathbf{v}}_{\mathrm{t}}$ and $\hat{\mathbf{i}}=\mathbf{B}^{T} \hat{\mathbf{i}}_{1}$, the reciprocity condition can be rewritten as

$$
\tilde{\mathbf{v}}_{\mathbf{t}}^{T} \mathbf{Q} \mathbf{B}^{T} \hat{\mathbf{i}}_{1}=\hat{\mathbf{v}}_{\mathbf{t}}^{T} \mathbf{Q} \mathbf{B}^{T} \tilde{\mathbf{i}}_{1}
$$

As condition (4) holds valid independently of the chosen set of signals $\left\{\tilde{\mathbf{v}}_{\mathrm{t}}, \tilde{\mathbf{i}}_{\mathrm{l}}, \hat{\mathbf{v}}_{\mathrm{t}}, \hat{\mathbf{i}}_{1}\right\}, \mathbf{Q}$ and $\mathbf{B}$ must satisfy the orthogonality property

$$
\mathbf{Q B}^{T}=\mathbf{0},
$$

where $\mathbf{0}$ is a zero matrix [6]. The orthogonality property (5) quite obviously makes equality (4) always satisfied. Equality (5) also implies

$$
\mathbf{B Q}^{T}=\mathbf{0}
$$

It is worth pointing out that the orthogonality properties (5) and (6) hold irrespectively of the ordering of port variables. In fact, the ordering used above is not a mandatory choice, but comes in handy when studying the general relation between $\mathbf{Q}$ and $\mathbf{B}$, as shown in what follows.

If we use eq. (2) in (5) or (6), we obtain

$$
\left[\begin{array}{ll}
\mathbf{Q}_{\mathrm{q}} & \mathbf{I}
\end{array}\right]\left[\begin{array}{c}
\mathbf{I} \\
\mathbf{B}_{\mathrm{p}}^{T}
\end{array}\right]=\mathbf{0},
$$

and we deduce that

$$
\mathbf{Q}_{\mathrm{q}}=-\mathbf{B}_{\mathrm{p}}^{T}
$$

It follows that, in the light of (8), eq. (2) can be rewritten as

$$
\mathbf{Q}=\left[\begin{array}{ll}
\mathbf{F} & \mathbf{I}
\end{array}\right] \quad, \quad \mathbf{B}=\left[\begin{array}{ll}
\mathbf{I} & -\mathbf{F}^{T}
\end{array}\right],
$$

where $\mathbf{Q}_{\mathrm{q}}=\mathbf{F}$ and $\mathbf{B}_{\mathrm{p}}=-\mathbf{F}^{T}$.

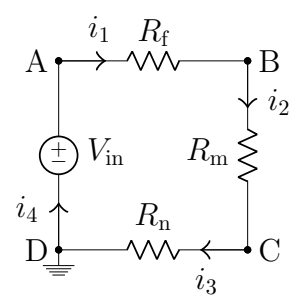

(a)

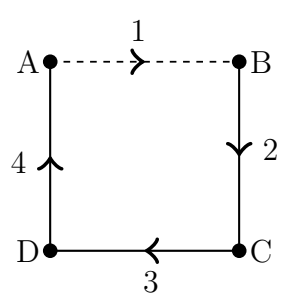

(b)
Fig. 1. Fig. 1(a) shows a circuit with 4 one-ports connected in series; Fig. 1(b) shows its digraph representation. The link of the cotree subgraph is a dashed line, the 3 twigs of the tree subgraph are continuous lines. 


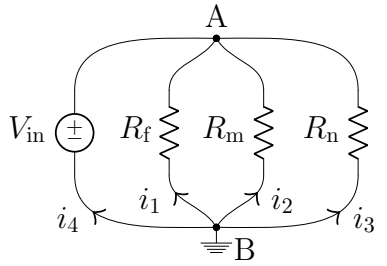

(a)

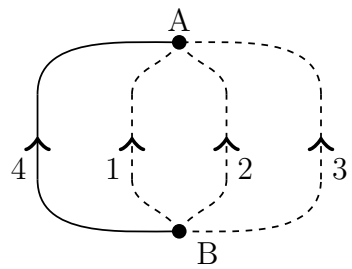

(b)
Fig. 2. Fig. 2(a) shows a circuit with 4 one-ports connected in parallel; Fig. 2(b) shows its digraph representation. The twig of the tree subgraph is a continuous line, the 3 links of the cotree subgraph are dashed lines.

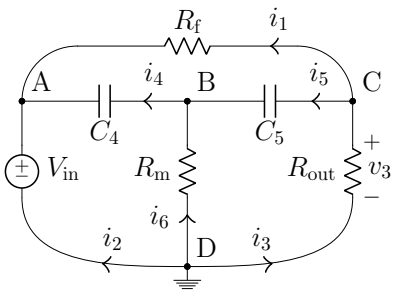

(a)

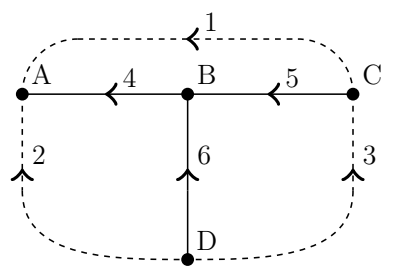

(b)
Fig. 3. Fig. 3(a) shows a circuit with 6 one-ports and bridged-T topology; Fig. 3(b) shows its digraph representation. The 3 twigs of the tree subgraph are continuous lines, the 3 links of the cotree subgraph are dashed lines.

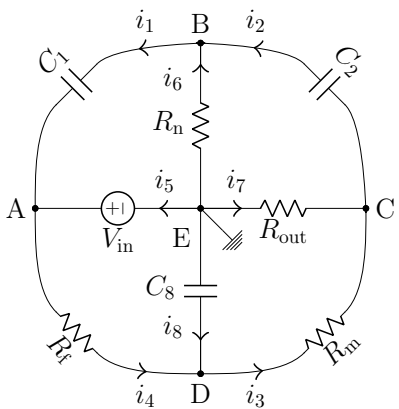

(a)

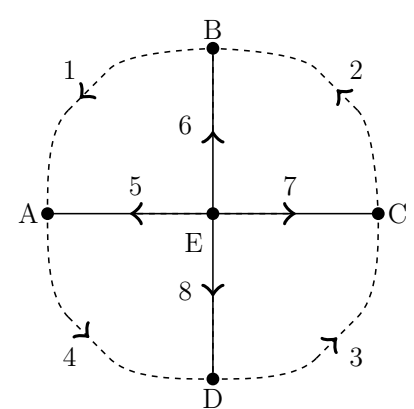

(b)
Fig. 4. Fig. 4(a) shows a circuit with 8 one-ports and twin-T topology; Fig. 4(b) shows its digraph representation. The 4 twigs of the tree subgraph are continuous line, the 4 links of the cotree subgraph are dashed lines.

\section{A. Connection Networks based on "Wire Interconnections"}

Topological connection networks solely based on "wire interconnections" are inherently reciprocal [26]. In such cases, the matrices $\mathbf{Q}$ and $\mathbf{B}$ are reduced to the well-known fundamental cut set matrix and fundamental loop matrix [25], respectively. Series and parallel topologies are the simplest and most widespread examples of the sort, but this class of connection networks also includes more complex topologies that are neither series nor parallel [27], [28]. The elegant tree-cotree decomposition method based on graph theory [1], [24] can be applied to connection networks of this class in order to identify suitable sets of independent port variables. According to the method, the reference $N$-port connection network is represented as a directed graph (digraph). The vertices of the digraph correspond to the nodes of the network, $N$ branches represent the arbitrary elements connected to the $N$ ports and the direction of the arrows on the branches is the same of port currents. The digraph is decomposed into two subgraphs: a tree, which is a connected subgraph without loops containing all nodes of the digraph, and a cotree, which is the complementary subgraph of the selected tree. Branches are then partitioned into tree branches, called twigs, and cotree branches, called links. Once the partition is performed, port voltages associated to twigs form the vector of independent port voltages $\mathbf{v}_{\mathrm{t}}$; while port currents associated to links form the vector of independent port currents $\mathbf{i}_{1}$.

We now discuss some examples of connection networks that are solely based on "wire interconnections", as well as the partition of the branches of the corresponding digraphs into twigs and links. In all the examples that follow, ports are numbered according to the convention used in (9).

1) Series Topology: The digraph corresponding to a $N$-port series connection network is characterized by $N-1$ twigs and 1 link. It follows that $q=N-1$ and $p=1$. The matrix $\mathbf{F}$ in (9) becomes $\mathbf{F}=-\mathbb{1}_{N-1}$, where $\mathbb{1}_{N-1}$ is a column vector of size $(N-1) \times 1$ with unitary entries. The vectors of independent port variables are $\mathbf{v}_{\mathbf{t}}=\left[v_{2}, \ldots, v_{N}\right]^{T}$ and $\mathbf{i}_{1}=i_{1}$. The circuit in Fig. 1(a) for instance, is characterized by the series connection network in Fig. 5(a) and its corresponding digraph is shown in Fig. 1(b)

2) Parallel Topology: The digraph corresponding to a $N$ port parallel connection network is the dual of the one representing a series topology and it is characterized by 1 twig and $N-1$ links. It follows that $q=1$ and $p=N-1$. Matrix $\mathbf{F}$ becomes $\mathbf{F}=\mathbb{1}_{N-1}^{T}, \mathbf{v}_{\mathrm{t}}=v_{N}$ and $\mathbf{i}_{1}=\left[i_{1}, \ldots, i_{N-1}\right]^{T}$. The circuit in Fig. 2(a) for instance, is characterized by the parallel connection network in Fig. 5(b) and its corresponding digraph is shown in Fig. 2(b)

3) Bridged-T Topology: The circuit in Fig. 3(a) is characterized by the 6-port bridged-T connection network [1] in Fig. 5(c), which is neither a series nor a parallel connection network. The corresponding digraph is shown in Fig. 3(b) where one possible tree with 3 twigs and the associated cotree with 3 links are highlighted. It follows that $p=q=3$. The matrix $\mathbf{F}$, needed for deriving $\mathbf{Q}$ and $\mathbf{B}$, is

$$
\mathbf{F}=\left[\begin{array}{ccc}
1 & 1 & 0 \\
1 & 0 & -1 \\
0 & 1 & 1
\end{array}\right]
$$

$\mathbf{v}_{\mathrm{t}}=\left[v_{4}, v_{5}, v_{6}\right]^{T}$ and $\mathbf{i}_{1}=\left[i_{1}, i_{2}, i_{3}\right]^{T}$.

4) Twin-T Topology: Another example of circuit characterized neither by a series nor by a parallel connection network is the one in Fig. 4(a) and its 8-port twin-T connection network [4] is shown in Fig. 5(d) The corresponding digraph is shown in Fig. 4(b), where one possible tree with 4 twigs and the associated cotree with 4 links are highlighted. It follows that $p=q=4$. The matrix $\mathbf{F}$, needed for deriving $\mathbf{Q}$ and $\mathbf{B}$, is

$$
\mathbf{F}=\left[\begin{array}{cccc}
1 & 0 & 0 & -1 \\
-1 & 1 & 0 & 0 \\
0 & -1 & 1 & 0 \\
0 & 0 & -1 & 1
\end{array}\right]
$$

$\mathbf{v}_{\mathbf{t}}=\left[v_{5}, v_{6}, v_{7}, v_{8}\right]^{T}$ and $\mathbf{i}_{1}=\left[i_{1}, i_{2}, i_{3}, i_{4}\right]^{T}$. 


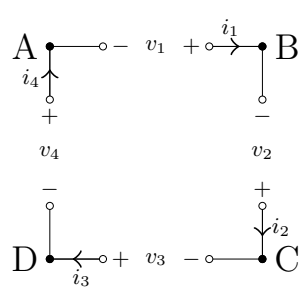

(a)

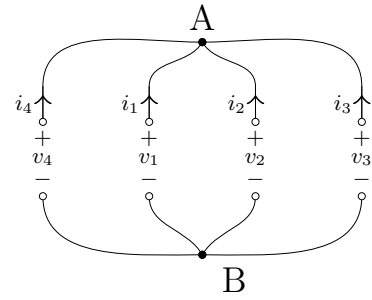

(b)

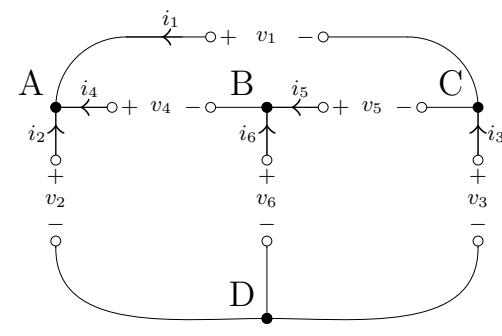

(c)

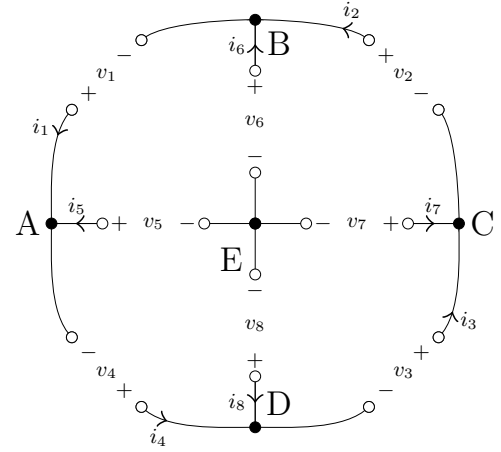

(d)

Fig. 5. Examples of connection networks based on wire interconnections. Fig. 5(a) Fig. 5(b) Fig. 5(c) Fig. 5(d) show a 4-port series connection network, a 4-port parallel connection network, a 6-port bridged-T connection network and a 8-port twin-T connection network, respectively.

\section{B. Connection Networks embedding Reciprocal Multi-ports}

Equations (1) are not only suitable for describing arbitrarily complex "purely topological" connection networks, i.e. solely based on "wire interconnections", but also more general connection networks embedding reciprocal multi-ports, such as ideal transformers [5]. In what follows we discuss some examples of connection networks made of "wire interconnections" and ideal transformers and we describe them in terms of independent port voltages and port currents, according to eqs. (1). In particular, a description of the sort is applied to $N$-port ideal multi-winding transformers.



(a)

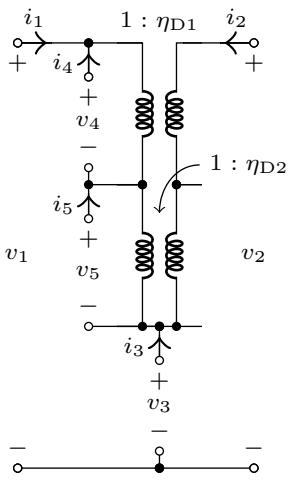

(b)
Fig. 6. Examples of connection networks embedding transformers. Fig. 6(a) and Fig. 6(b) show the 4-port connection network of a Brune section and the 5 -port connection network of a Darlington-D section, respectively.

1) Brune Section: Fig. 6(a) shows the 4-port connection network of the so-called Brune section [6], [29]. It can be verified that $p=2$ and $q=2$. Defining $\mathbf{v}_{\mathrm{t}}=\left[v_{3}, v_{4}\right]^{T}$ and $\mathbf{i}_{1}=\left[i_{1}, i_{2}\right]^{T}$, the resulting $\mathbf{F}$ matrix is

$$
\mathbf{F}=\left[\begin{array}{cc}
1 & \eta_{\mathrm{B}} \\
1 & 1
\end{array}\right],
$$

where $\eta_{\mathrm{B}}$ is the turns ratio of the ideal transformer. It follows that $p=q=2$.

2) Darlington-D Section: Fig. 6(b) shows the 5-port connection network of the so-called Darlington-D section [30], [31]. It can be verified that $q=3$ and $p=2$. Defining

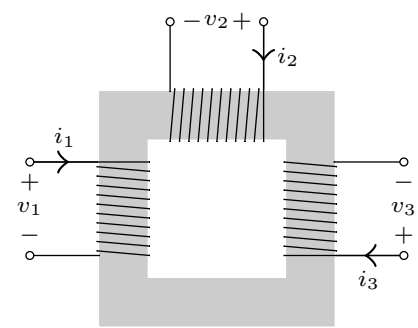

(a)

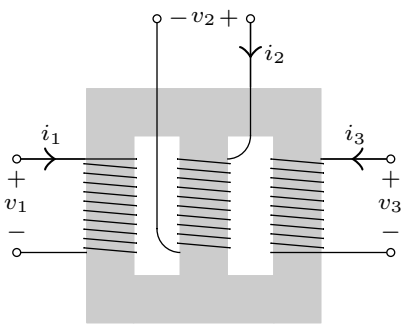

(b)
Fig. 7. Examples of multi-winding transformers. Fig. 7(a) and Fig. 7(b) show the 3-port connection network of a 3-winding transformer with series structure and the 3-port connection network of a 3-winding transformer with parallel structure, respectively.

$\mathbf{v}_{\mathbf{t}}=\left[v_{3}, v_{4}, v_{5}\right]^{T}$ and $\mathbf{i}_{1}=\left[i_{1}, i_{2}\right]^{T}$, the resulting $\mathbf{F}$ matrix is

$$
\mathbf{F}=\left[\begin{array}{cc}
1 & 1 \\
1 & \eta_{\mathrm{D} 1} \\
1 & \eta_{\mathrm{D} 2}
\end{array}\right],
$$

where $\eta_{\mathrm{D} 1}$ and $\eta_{\mathrm{D} 2}$ are the turns ratios of the two ideal transformers.

3) Ideal Multi-winding Transformer with Series Structure: Let us consider an $N$-port ideal $N$-winding transformer characterized by a series magnetic structure. Fig. 7(a) shows an example of a 3 -winding transformer of the sort. $\gamma_{1}, \ldots, \gamma_{N}$ are the numbers of turns of the $N$ windings, respectively. Port voltages are related by the constraint

$$
v_{1} / \gamma_{1}=v_{2} / \gamma_{2}=\cdots=v_{N} / \gamma_{N},
$$

while port currents satisfy the equation

$$
\gamma_{1} i_{1}+\gamma_{2} i_{2}+\cdots+\gamma_{N} i_{N}=0 \text {. }
$$

As each port voltage $v_{n}$ with $1 \leq n \leq N$ can be expressed as $v_{n}=\left(\gamma_{n} / \gamma_{N}\right) v_{N}$, we deduce that $q=1$ and $p=N-1$. It follows that

$$
\mathbf{F}=\left[\gamma_{1} / \gamma_{N}, \gamma_{2} / \gamma_{N}, \ldots, \gamma_{N-1} / \gamma_{N}\right]
$$

It is worth noticing that an ideal $N$-winding transformer with series structure can be interpreted as a generalization of a connection network with parallel topology; in fact an $N$-port 
parallel junction is like an ideal $N$-winding transformer with series structure where $\gamma_{n}=\gamma_{N}, \forall n \in\{1,2, \ldots, N\}$. This is in accordance with the duality principles relating electric and magnetic circuits discussed in [32].

4) Ideal Multi-winding Transformer with Parallel Structure: Let us now consider a $N$-port ideal $N$-winding transformer characterized by a parallel magnetic structure. Fig. $7(\mathrm{~b})$ shows an example of a 3 -winding transformer of the sort. $\gamma_{1}, \ldots, \gamma_{N}$ are the numbers of turns of the $N$ windings, respectively. Port currents are related by the constraint

$$
\gamma_{1} i_{1}=\gamma_{2} i_{2}=\cdots=\gamma_{N} i_{N},
$$

while port voltages obey the equation

$$
v_{1} / \gamma_{1}+v_{2} / \gamma_{2}+\cdots+v_{N} / \gamma_{N}=0 .
$$

It follows that matrix $\mathbf{F}$ is given by

$$
\mathbf{F}=-\left[\gamma_{1} / \gamma_{2}, \gamma_{1} / \gamma_{3}, \ldots, \gamma_{1} / \gamma_{N}\right]^{T} .
$$

It is worth noticing that an ideal $N$-winding transformer with parallel structure can be interpreted as a generalization of a connection network with series topology; in fact an $\mathrm{N}$-port series junction is like an ideal $N$-winding transformer with parallel structure where $\gamma_{n}=\gamma_{1}, \forall n \in\{1,2, \ldots, N\}$.

\section{ReCIPROCAL JUNCTIONS IN GENERALIZED WDFs}

The previous Section discussed the general properties of $N$-port reciprocal connection networks in the domain of port voltages and port currents, along with some examples. This Section shows how the same reciprocal connection networks can be represented in the WD domain using scattering junctions, characterized by scattering matrices. Scattering matrices will be derived and their properties will be discussed, starting from a generalized definition of wave signals that includes traditional definitions of voltage waves, current waves and power-normalized waves as particular cases.

\section{A. Generalized Definition of Wave Signals}

The generalized definition of wave port variables in WDFs, recently proposed in [7], [12], is the following

$$
\mathbf{a}=\mathbf{R}^{\rho-1} \mathbf{v}+\mathbf{R}^{\rho} \mathbf{i}, \mathbf{b}=\mathbf{R}^{\rho-1} \mathbf{v}-\mathbf{R}^{\rho} \mathbf{i},
$$

where $\mathbf{a}=\left[a_{1}, \ldots, a_{N}\right]^{T}$ and $\mathbf{b}=\left[b_{1}, \ldots, b_{N}\right]^{T}$ are the vectors of so called incident and reflected waves, $\mathbf{R}=$ $\operatorname{diag}\left[R_{1}, \ldots, R_{N}\right]$ is a diagonal matrix of free parameters called port resistances, and $\rho$ is a real parameter. Setting $\rho=1$, 20 reduces to the traditional definition of voltage waves proposed by Fettweis in [2]; setting $\rho=0$, 20] reduces to the dual definition of current waves; while, setting $\rho=1 / 2$, (20) reduces to the definition of power-normalized waves. Infinite other choices of $\rho$ are possible, leading to infinite new definitions of wave signals with hybrid units of measure. The inverse mapping of 20$]$ is the following

$$
\mathbf{v}=\frac{1}{2} \mathbf{R}^{1-\rho}(\mathbf{a}+\mathbf{b}), \mathbf{i}=\frac{1}{2} \mathbf{R}^{-\rho}(\mathbf{a}-\mathbf{b}) .
$$

TABLE I

Generalized WDF SCATTERING Matrix PROPERTIES

\begin{tabular}{c|c}
\hline Name & Equation \\
\hline Losslessness & $\mathbf{S}^{T} \mathbf{R}^{1-2 \rho} \mathbf{S}=\mathbf{R}^{1-2 \rho}$ \\
\hline Self-inverse property & $\mathbf{S S}=\mathbf{I}$ \\
\hline Reciprocity & $\mathbf{S}^{T} \mathbf{R}^{1-2 \rho}=\mathbf{R}^{1-2 \rho} \mathbf{S}$ \\
\hline
\end{tabular}

\section{B. Scattering Matrix Properties}

The $N$-port WD junction representing an arbitrary $N$-port reference connection network is characterized by a scattering matrix $\mathbf{S}$, which relates the vector a of waves incident to the junction to the vector $\mathbf{b}$ of reflected waves as follows

$$
\mathbf{b}=\mathbf{S a} \text {. }
$$

The three main properties of $\mathbf{S}$, i.e., losslessness, self-inverse property and reciprocity, are summarized in Table II and discussed in the following.

1) Losslessness: As every connection network is lossless, the power $W$ absorbed by the $N$-port is zero, therefore we can write

$$
W=\mathbf{v}^{T} \mathbf{i}=0 .
$$

It follows that, according to equations 23, 21) and 22,

$$
W=\frac{1}{4} \mathbf{a}^{T}\left(\mathbf{R}^{1-2 \rho}-\mathbf{S}^{T} \mathbf{R}^{1-2 \rho} \mathbf{S}\right) \mathbf{a}=\mathbf{0} .
$$

From 24, we derive the losslessness property

$$
\mathbf{S}^{T} \mathbf{R}^{1-2 \rho} \mathbf{S}=\mathbf{R}^{1-2 \rho},
$$

since $W=0$ must be satisfied independently of a.

2) Self-inverse property: Similarly to what pointed out in [33] for voltage waves; as the vectors $\mathbf{v}$ and $\mathbf{i}$ are solutions of independent homogeneous linear equations, from $\mathbf{b}=\mathrm{Sa}$, we deduce

$$
\xi \mathbf{R}^{\rho-1} \mathbf{v}-\zeta \mathbf{R}^{\rho} \mathbf{i}=\mathbf{S}\left(\xi \mathbf{R}^{\rho-1} \mathbf{v}+\zeta \mathbf{R}^{\rho} \mathbf{i}\right),
$$

where $\xi$ and $\zeta$ are arbitrary real numbers. Therefore, if we choose $\xi=-1$ and $\zeta=1$ the inverse mapping $\mathbf{a}=\mathbf{S b}$ must hold true as well. This means that $\mathbf{S}$ is an involutory matrix and the following self-inverse property must be satisfied

$$
\mathbf{S S}=\mathbf{I}
$$

3) Reciprocity: Combining equations 25) and 27, we deduce the following reciprocity property of $\mathbf{S}$

$$
\mathbf{S}^{T} \mathbf{R}^{1-2 \rho}=\mathbf{R}^{1-2 \rho} \mathbf{S} .
$$

In fact, we will show in the following that the reciprocity condition (3), involving the port variables of the reference connection network, implies the property (28). First, we rewrite the reciprocity condition $(3)$ in the WD domain as

$$
\left(\tilde{\mathbf{a}}^{T}-\tilde{\mathbf{b}}^{T}\right) \mathbf{R}^{1-2 \rho}(\hat{\mathbf{a}}-\hat{\mathbf{b}})=\left(\hat{\mathbf{a}}^{T}-\hat{\mathbf{b}}^{T}\right) \mathbf{R}^{1-2 \rho}(\tilde{\mathbf{a}}-\tilde{\mathbf{b}})
$$

where the two pairs of vectors of wave signals

$$
\begin{aligned}
& \left\{\tilde{\mathbf{a}}=\left[\tilde{a}_{1}, \ldots, \tilde{a}_{N}\right]^{T}, \tilde{\mathbf{b}}=\left[\tilde{b}_{1}, \ldots, \tilde{b}_{N}\right]^{T}\right\}, \\
& \left\{\hat{\mathbf{a}}=\left[\hat{a}_{1}, \ldots, \hat{a}_{N}\right]^{T}, \hat{\mathbf{b}}=\left[\hat{b}_{1}, \ldots, \hat{b}_{N}\right]^{T}\right\},
\end{aligned}
$$


are obtained from the two pairs of vectors $\{\tilde{\mathbf{v}}, \tilde{\mathbf{i}}\}$ and $\{\hat{\mathbf{v}}, \hat{\mathbf{i}}\}$, after applying the transformation (21) two times with the same diagonal matrix of port resistances $\mathbf{R}$ and parameter $\rho$. As $\tilde{\mathbf{b}}=$ Sa and $\hat{\mathbf{b}}=$ Sâ, 29 can be written as

$$
\tilde{\mathbf{a}}^{T}\left(\mathbf{I}+\mathbf{S}^{T}\right) \mathbf{R}^{1-2 \rho}(\mathbf{I}-\mathbf{S}) \hat{\mathbf{a}}=\hat{\mathbf{a}}^{T}\left(\mathbf{I}+\mathbf{S}^{T}\right) \mathbf{R}^{1-2 \rho}(\mathbf{I}-\mathbf{S}) \tilde{\mathbf{a}} .
$$

Condition (32) is satisfied for any pair of vectors of incident waves ã and $\hat{\mathbf{a}}$, when

$$
\left(\mathbf{I}+\mathbf{S}^{T}\right) \mathbf{R}^{1-2 \rho}(\mathbf{I}-\mathbf{S})=\mathbf{0}
$$

or, equivalently, when

$$
\mathbf{R}^{1-2 \rho}-\mathbf{R}^{1-2 \rho} \mathbf{S}+\mathbf{S}^{T} \mathbf{R}^{1-2 \rho}-\mathbf{S}^{T} \mathbf{R}^{1-2 \rho} \mathbf{S}=\mathbf{0} .
$$

Condition (34) is always verified only if both the losslessness property (25) and the reciprocity property (28) hold true.

4) Power-normalized case: In the special case in which power-normalized waves are used, $\mathbf{S}$ becomes an orthogonal matrix. In fact, by replacing $\rho=1 / 2$ into $(28)$, we obtain

$$
\mathbf{S}=\mathbf{S}^{T},
$$

therefore $\mathbf{S}$ is a symmetric matrix. Moreover, by combining (35) with (27), we obtain

$$
\mathbf{S}^{T}=\mathbf{S}^{-1}
$$

therefore, $\mathbf{S}$ is orthogonal.

\section{Scattering Matrix Derivation}

By replacing (1) into the generalized definition of wave signals 20, we derive

$$
\begin{aligned}
& \mathbf{a}=\mathbf{R}^{\rho-1} \mathbf{Q}^{T} \mathbf{v}_{\mathbf{t}}+\mathbf{R}^{\rho} \mathbf{B}^{T} \mathbf{i}_{1} \\
& \mathbf{b}=\mathbf{R}^{\rho-1} \mathbf{Q}^{T} \mathbf{v}_{\mathbf{t}}-\mathbf{R}^{\rho} \mathbf{B}^{T} \mathbf{i}_{1}
\end{aligned}
$$

and by left-multiplying both sides of eq. (37) by $\mathbf{Q R} \mathbf{R}^{-\rho}$, we obtain

$$
\mathbf{Q R}^{-\rho} \mathbf{a}=\mathbf{Q R}^{-1} \mathbf{Q}^{T} \mathbf{v}_{\mathbf{t}} .
$$

Similarly, by left-multiplying both sides of eq. (38) by $\mathbf{B R}^{1-\rho}$, after simplifying the resulting expression we obtain

$$
\mathbf{B R}^{1-\rho} \mathbf{b}=-\mathbf{B R B}^{T} \mathbf{i}_{1} .
$$

By solving (39) and (40) for $\mathbf{v}_{\mathbf{t}}$ and $\mathbf{i}_{1}$, respectively, we obtain

$$
\begin{aligned}
& \mathbf{v}_{\mathbf{t}}=\left(\mathbf{Q R}^{-1} \mathbf{Q}^{T}\right)^{-1} \mathbf{Q} \mathbf{R}^{-\rho} \mathbf{a} \\
& \mathbf{i}_{1}=-\left(\mathbf{B R B}^{T}\right)^{-1} \mathbf{B} \mathbf{R}^{1-\rho} \mathbf{b}
\end{aligned}
$$

In order to compact the expressions, we now define

$$
\mathbf{\Psi}=\mathbf{Q}^{T}\left(\mathbf{Q R}^{-1} \mathbf{Q}^{T}\right)^{-1} \mathbf{Q} \text { and } \boldsymbol{\Lambda}=\mathbf{B}^{T}\left(\mathbf{B R B}^{T}\right)^{-1} \mathbf{B} \text {, }
$$

so that, after plugging both (41) and (42) in (37) and in (38), we can write

$$
\begin{aligned}
\mathbf{a} & =\mathbf{R}^{\rho-1} \mathbf{\Psi} \mathbf{R}^{-\rho} \mathbf{a}-\mathbf{R}^{\rho} \boldsymbol{\Lambda} \mathbf{R}^{1-\rho} \mathbf{b} \\
\mathbf{b} & =\mathbf{R}^{\rho-1} \mathbf{\Psi} \mathbf{R}^{-\rho} \mathbf{a}+\mathbf{R}^{\rho} \boldsymbol{\Lambda} \mathbf{R}^{1-\rho} \mathbf{b}
\end{aligned}
$$

Adding eqs. (44) and 45) together and solving for $\mathbf{b}$ yields

$$
\mathbf{b}=2 \mathbf{R}^{\rho-1} \mathbf{\Psi} \mathbf{R}^{-\rho} \mathbf{a}-\mathbf{a} .
$$

Therefore, the scattering matrix $\mathbf{S}$ can be expressed as

$$
\mathbf{S}=2 \mathbf{R}^{\rho-1} \mathbf{\Psi} \mathbf{R}^{-\rho}-\mathbf{I} .
$$

Subtracting eq. 444 from eq. (45) and solving for a returns

$$
\mathbf{a}=\left(\mathbf{I}-2 \mathbf{R}^{\rho} \boldsymbol{\Lambda} \mathbf{R}^{1-\rho}\right) \mathbf{b},
$$

which, exploiting the self-inverse property (27), allows us to derive the following alternative expression for $\mathbf{S}$

$$
\mathbf{S}=\mathbf{I}-2 \mathbf{R}^{\rho} \boldsymbol{\Lambda} \mathbf{R}^{1-\rho} .
$$

It is worth noticing that the most computationally demanding operation to derive $\mathbf{S}$ is the inversion of a linear system of equation. When using eq. (47), this corresponds to inverting the $q \times q$ matrix $\mathbf{Q R}^{-1} \mathbf{Q}^{T}$ in order to compute $\boldsymbol{\Psi}$. When using eq. (49), on the other hand, we need to invert the $p \times p$ matrix $\mathbf{B R B}^{T}$ to compute $\boldsymbol{\Lambda}$. As $q$ is generally different from $p$, it might be computationally advantageous to carefully select one of the two eqs. (47) and 497.

\section{RECIPROCAL JUNCTIONS IN BIPARAMETRIC WDFS}

In this Section, we consider a family of WD structures, recently introduced in [23], characterized by wave definitions involving two free parameters per port instead of one and known as Biparametric WDFs (BWDFs). We restate in a more general fashion the pair of "dual" biparametric definitions of wave signals originally introduced in [23] and we derive the corresponding WD junctions, which describe arbitrary reciprocal $N$-port connection networks.

\section{A. Biparametric Definition of Waves}

We first consider the following definition of wave variables

$$
\mathbf{a}=\mu \boldsymbol{\Phi}^{-1}\left(\mathbf{v}+\mathbf{R}_{\beta} \mathbf{i}\right), \quad \mathbf{b}=\mu \boldsymbol{\Phi}^{-1}\left(\mathbf{v}-\mathbf{R}_{\alpha} \mathbf{i}\right),
$$

where vectors $\mathbf{v}, \mathbf{i}, \mathbf{a}$ and $\mathbf{b}$ are defined as in Section III $\mu$ is an arbitrary dimension-less constant (set equal to $1 / 2$ in [23]), $\mathbf{R}_{\alpha}=\operatorname{diag}\left[\alpha_{1}, \ldots, \alpha_{N}\right]$ and $\mathbf{R}_{\beta}=\operatorname{diag}\left[\beta_{1}, \ldots, \beta_{N}\right]$, being $\alpha_{n}$ and $\beta_{n}$, with $1 \leq n \leq N$, positive real free parameters having the same unit of measurement of resistances. In the case of power-normalized waves [23] we have $\boldsymbol{\Phi}=\operatorname{diag}\left[\varphi_{1}, \ldots, \varphi_{N}\right]$ with $\varphi_{n}=\sqrt{\left(\alpha_{n}+\beta_{n}\right) / 2 \text {. It is worth }}$ noticing that, in the special case of $\mathbf{R}=\mathbf{R}_{\alpha}=\mathbf{R}_{\beta}(\rho=1 / 2$ and $\mu=1$ ), (50) is equivalent to (20); therefore, (50) can be seen as a generalization of the traditional definition of powernormalized waves. Other biparametric definitions of waves with different units of measurement (e.g., voltage waves or current waves) can be derived through alternative definitions of the normalization matrix $\boldsymbol{\Phi}$. An in-depth discussion of such cases, however, will not be presented here for the sake of simplicity. The inverse mapping of 50 is given by

$$
\begin{array}{r}
\mathbf{v}=\frac{1}{\mu} \boldsymbol{\Phi}\left(\mathbf{R}_{\alpha}+\mathbf{R}_{\beta}\right)^{-1}\left(\mathbf{R}_{\alpha} \mathbf{a}+\mathbf{R}_{\beta} \mathbf{b}\right) \\
\mathbf{i}=\frac{1}{\mu} \boldsymbol{\Phi}\left(\mathbf{R}_{\alpha}+\mathbf{R}_{\beta}\right)^{-1}(\mathbf{a}-\mathbf{b})
\end{array}
$$

We call $\mathbf{S}_{\varphi}$ the scattering matrix describing the reference connection network, so that $\mathbf{b}=\mathbf{S}_{\varphi} \mathbf{a}$; in the following, we will show four possible ways of deriving $\mathbf{S}_{\varphi}$. 
By replacing (1) into (50), we obtain

$$
\begin{aligned}
& \mathbf{a}=\mu \boldsymbol{\Phi}^{-1}\left(\mathbf{Q}^{T} \mathbf{v}_{\mathbf{t}}+\mathbf{R}_{\beta} \mathbf{B}^{T} \mathbf{i}_{1}\right) \\
& \mathbf{b}=\mu \boldsymbol{\Phi}^{-1}\left(\mathbf{Q}^{T} \mathbf{v}_{\mathbf{t}}-\mathbf{R}_{\alpha} \mathbf{B}^{T} \mathbf{i}_{\mathbf{l}}\right)
\end{aligned}
$$

Left-multiplying both sides of eq. 53 by $\mathbf{Q} \mathbf{\Phi} \mathbf{R}_{\beta}^{-1}$ yields

$$
\mathbf{Q} \Phi \mathbf{R}_{\beta}^{-1} \mathbf{a}=\mu \mathbf{Q R} \mathbf{R}_{\beta}^{-1} \mathbf{Q}^{T} \mathbf{v}_{\mathbf{t}} .
$$

By left-multiplying both sides of eq. 54 by $\mathbf{B} \boldsymbol{\Phi}$, we obtain

$$
\mathbf{B} \mathbf{\Phi} \mathbf{b}=-\mu \mathbf{B} \mathbf{R}_{\alpha} \mathbf{B}^{T} \mathbf{i}_{1} .
$$

Solving (55) and (56) for $\mathbf{v}_{\mathrm{t}}$ and $\mathbf{i}_{1}$, respectively, returns

$$
\begin{array}{r}
\mathbf{v}_{\mathbf{t}}=\frac{1}{\mu}\left(\mathbf{Q R}_{\beta}^{-1} \mathbf{Q}^{T}\right)^{-1} \mathbf{Q} \mathbf{\Phi} \mathbf{R}_{\beta}^{-1} \mathbf{a} \\
\mathbf{i}_{1}=-\frac{1}{\mu}\left(\mathbf{B} \mathbf{R}_{\alpha} \mathbf{B}^{T}\right)^{-1} \mathbf{B} \mathbf{\Phi} \mathbf{b}
\end{array}
$$

We can now compact the expressions by defining

$$
\boldsymbol{\Psi}_{\beta}=\mathbf{Q}^{T}\left(\mathbf{Q R}_{\beta}^{-1} \mathbf{Q}^{T}\right)^{-1} \mathbf{Q}, \quad \boldsymbol{\Lambda}_{\alpha}=\mathbf{B}^{T}\left(\mathbf{B R}_{\alpha} \mathbf{B}^{T}\right)^{-1} \mathbf{B},
$$

so that, after replacing both (57) and (58) in eqs. (53) and (54), we can write

$$
\begin{aligned}
\mathbf{a} & =\boldsymbol{\Phi}^{-1} \boldsymbol{\Psi}_{\beta} \boldsymbol{\Phi} \mathbf{R}_{\beta}^{-1} \mathbf{a}-\mathbf{R}_{\beta} \boldsymbol{\Phi}^{-1} \boldsymbol{\Lambda}_{\alpha} \boldsymbol{\Phi} \mathbf{b} \\
\mathbf{b} & =\boldsymbol{\Phi}^{-1} \boldsymbol{\Psi}_{\beta} \boldsymbol{\Phi} \mathbf{R}_{\beta}^{-1} \mathbf{a}+\mathbf{R}_{\alpha} \boldsymbol{\Phi}^{-1} \boldsymbol{\Lambda}_{\alpha} \boldsymbol{\Phi} \mathbf{b}
\end{aligned}
$$

By left-multiplying both sides of eq. 60 by $\mathbf{R}_{\beta}^{-1}$, leftmultiplying both sides of eq. (61) by $\mathbf{R}_{\alpha}^{-1}$, adding together the two resulting equations and solving for $\mathbf{b}$, we obtain

$$
\mathbf{b}=\left(\mathbf{R}_{\alpha}\left(\mathbf{R}_{\alpha}^{-1}+\mathbf{R}_{\beta}^{-1}\right) \boldsymbol{\Phi}^{-1} \mathbf{\Psi}_{\beta} \mathbf{\Phi} \mathbf{R}_{\beta}^{-1}-\mathbf{R}_{\alpha} \mathbf{R}_{\beta}^{-1}\right) \mathbf{a} .
$$

Subtracting eq. 61) from eq. 600 and solving for a, yields

$$
\mathbf{a}=\left(\mathbf{I}-\left(\mathbf{R}_{\alpha}+\mathbf{R}_{\beta}\right) \boldsymbol{\Phi}^{-1} \boldsymbol{\Lambda}_{\alpha} \boldsymbol{\Phi}\right) \mathbf{b} .
$$

Alternatively, by left-multiplying both sides of eq. 53 by $\mathbf{B} \boldsymbol{\Phi}$ and both sides of eq. (54) by $\mathbf{Q} \boldsymbol{\Phi} \mathbf{R}_{\alpha}^{-1}$ and, by solving the two resulting equations for $\mathbf{i}_{1}$ and $\mathbf{v}_{t}$, respectively, we obtain

$$
\begin{array}{r}
\mathbf{i}_{1}=\frac{1}{\mu}\left(\mathbf{B R}_{\beta} \mathbf{B}^{T}\right)^{-1} \mathbf{B} \mathbf{\Phi} \mathbf{a} \\
\mathbf{v}_{\mathbf{t}}=\frac{1}{\mu}\left(\mathbf{Q R}_{\alpha}^{-1} \mathbf{Q}^{T}\right) \mathbf{Q} \mathbf{\Phi} \mathbf{R}_{\alpha}^{-1} \mathbf{b}
\end{array}
$$

We can now compact the expressions by defining

$$
\Psi_{\alpha}=\mathbf{Q}^{T}\left(\mathbf{Q R}_{\alpha}^{-1} \mathbf{Q}^{T}\right)^{-1} \mathbf{Q}, \quad \boldsymbol{\Lambda}_{\beta}=\mathbf{B}^{T}\left(\mathbf{B R}_{\beta} \mathbf{B}^{T}\right)^{-1} \mathbf{B},
$$

so that, after replacing both (64) and 65) in eqs. (53) and (54), we can write

$$
\begin{aligned}
& \mathbf{a}=\boldsymbol{\Phi}^{-1} \boldsymbol{\Psi}_{\alpha} \boldsymbol{\Phi} \mathbf{R}_{\alpha}^{-1} \mathbf{b}+\mathbf{R}_{\beta} \boldsymbol{\Phi}^{-1} \Lambda_{\beta} \boldsymbol{\Phi} \mathbf{a} \\
& \mathbf{b}=\boldsymbol{\Phi}^{-1} \boldsymbol{\Psi}_{\alpha} \boldsymbol{\Phi} \mathbf{R}_{\alpha}^{-1} \mathbf{b}-\mathbf{R}_{\alpha} \boldsymbol{\Phi}^{-1} \Lambda_{\beta} \boldsymbol{\Phi} \mathbf{a}
\end{aligned}
$$

By subtracting eq. (68) from eq. (67) and solving for $\mathbf{b}$, we obtain

$$
\mathbf{b}=\left(\mathbf{I}-\left(\mathbf{R}_{\alpha}+\mathbf{R}_{\beta}\right) \boldsymbol{\Phi}^{-1} \boldsymbol{\Lambda}_{\beta} \boldsymbol{\Phi}\right) \mathbf{a} .
$$

Left-multiplying both sides of eq. (67) by $\mathbf{R}_{\beta}^{-1}$, leftmultiplying both sides of eq. (68) by $\mathbf{R}_{\alpha}^{-1}$, summing the resulting equations and solving for $\mathbf{a}$, yields

$$
\mathbf{a}=\left(\mathbf{R}_{\beta}\left(\mathbf{R}_{\alpha}^{-1}+\mathbf{R}_{\beta}^{-1}\right) \boldsymbol{\Phi}^{-1} \boldsymbol{\Psi}_{\alpha} \mathbf{\Phi} \mathbf{R}_{\alpha}^{-1}-\mathbf{R}_{\beta} \mathbf{R}_{\alpha}^{-1}\right) \mathbf{b} .
$$

From eqs. 62), 69, (70), and 63), respectively, the following four equivalent expressions for the scattering matrix $\mathbf{S}_{\varphi}$ can be easily derived:

$$
\begin{aligned}
& \mathbf{S}_{\varphi}=\boldsymbol{\Phi}^{-1} \mathbf{R}_{\alpha}\left(\left(\mathbf{R}_{\alpha}^{-1}+\mathbf{R}_{\beta}^{-1}\right) \boldsymbol{\Psi}_{\beta}-\mathbf{I}\right) \mathbf{R}_{\beta}^{-1} \boldsymbol{\Phi} \\
& \mathbf{S}_{\varphi}=\boldsymbol{\Phi}^{-1}\left(\mathbf{I}-\left(\mathbf{R}_{\alpha}+\mathbf{R}_{\beta}\right) \boldsymbol{\Lambda}_{\beta}\right) \boldsymbol{\Phi} \\
& \mathbf{S}_{\varphi}=\left(\boldsymbol{\Phi}^{-1} \mathbf{R}_{\beta}\left(\left(\mathbf{R}_{\alpha}^{-1}+\mathbf{R}_{\beta}^{-1}\right) \boldsymbol{\Psi}_{\alpha}-\mathbf{I}\right) \mathbf{R}_{\alpha}^{-1} \boldsymbol{\Phi}\right)^{-1}(73) \\
& \mathbf{S}_{\varphi}=\left(\boldsymbol{\Phi}^{-1}\left(\mathbf{I}-\left(\mathbf{R}_{\alpha}+\mathbf{R}_{\beta}\right) \boldsymbol{\Lambda}_{\alpha}\right) \boldsymbol{\Phi}\right)^{-1}
\end{aligned}
$$

It should be quite clear that 71 and 72 are computationally less expensive than (73) and (74), which are only reported here for reasons of completeness.

\section{B. Dual Biparametric Definition of Waves}

Let us now consider the following dual definition of wave variables

$$
\mathbf{a}=\mu \boldsymbol{\Theta}^{-1}\left(\mathbf{R}_{\beta}^{-1} \mathbf{v}+\mathbf{i}\right), \quad \mathbf{b}=\mu \boldsymbol{\Theta}^{-1}\left(\mathbf{R}_{\alpha}^{-1} \mathbf{v}-\mathbf{i}\right)
$$

where $\mathbf{v}, \mathbf{i}, \mathbf{a}, \mathbf{b}, \mathbf{R}_{\alpha} \mathbf{R}_{\beta}$ and $\mu$ are defined as in the previous Subsection. In the case of power-normalized waves [23], we have $\boldsymbol{\Theta}=\operatorname{diag}\left[\theta_{1}, \ldots, \theta_{N}\right]$ with $\theta_{n}=\sqrt{\left(1 / \alpha_{n}+1 / \beta_{n}\right) / 2}$ and $\mu=1 / 2$. It is worth noticing that, even (75) can be seen as a generalization of the traditional definition of powernormalized waves; in fact, setting $\mathbf{R}=\mathbf{R}_{\alpha}=\mathbf{R}_{\beta}, \rho=1 / 2$ and $\mu=1$, (75) is equivalent to (20). The inverse mapping of (75) is given by eqs. 766 and 777.

$$
\begin{array}{r}
\mathbf{v}=\frac{1}{\mu} \boldsymbol{\Theta}\left(\mathbf{R}_{\alpha}^{-1}+\mathbf{R}_{\beta}^{-1}\right)^{-1}(\mathbf{a}+\mathbf{b}) \\
\mathbf{i}=\frac{1}{\mu} \boldsymbol{\Theta}\left(\mathbf{R}_{\alpha}^{-1}+\mathbf{R}_{\beta}^{-1}\right)^{-1}\left(\mathbf{R}_{\alpha}^{-1} \mathbf{a}-\mathbf{R}_{\beta}^{-1} \mathbf{b}\right)
\end{array}
$$

We call $\mathbf{S}_{\theta}$ the scattering matrix describing the reference connection network, so that $\mathbf{b}=\mathbf{S}_{\theta} \mathbf{a}$. Using a derivation method similar to the one described in the previous Subsection the following four equivalent expressions for the scattering matrix $\mathbf{S}_{\theta}$ are derived

$$
\begin{aligned}
& \mathbf{S}_{\theta}=\boldsymbol{\Theta}^{-1}\left(\left(\mathbf{R}_{\alpha}^{-1}+\mathbf{R}_{\beta}^{-1}\right) \mathbf{\Psi}_{\beta}-\mathbf{I}\right) \boldsymbol{\Theta} \\
& \mathbf{S}_{\theta}=\boldsymbol{\Theta}^{-1} \mathbf{R}_{\alpha}^{-1}\left(\mathbf{I}-\left(\mathbf{R}_{\alpha}+\mathbf{R}_{\beta}\right) \boldsymbol{\Lambda}_{\beta}\right) \mathbf{R}_{\beta} \boldsymbol{\Theta} \\
& \mathbf{S}_{\theta}=\left(\boldsymbol{\Theta}^{-1}\left(\left(\mathbf{R}_{\alpha}^{-1}+\mathbf{R}_{\beta}^{-1}\right) \mathbf{\Psi}_{\alpha}-\mathbf{I}\right) \boldsymbol{\Theta}\right)^{-1} \\
& \mathbf{S}_{\theta}=\left(\boldsymbol{\Theta}^{-1} \mathbf{R}_{\beta}^{-1}\left(\mathbf{I}-\left(\mathbf{R}_{\alpha}+\mathbf{R}_{\beta}\right) \boldsymbol{\Lambda}_{\alpha}\right) \mathbf{R}_{\alpha} \boldsymbol{\Theta}\right)^{-1}
\end{aligned}
$$

where $\boldsymbol{\Psi}_{\beta}, \boldsymbol{\Lambda}_{\beta}, \boldsymbol{\Psi}_{\alpha}$ and $\boldsymbol{\Lambda}_{\alpha}$ are defined as in (59) and (66).

\section{Properties of Scattering Matrices}

As far as self-inverse property is concerned, it can be verified that, when $\alpha_{n} \neq \beta_{n}$ with $1 \leq n \leq N, \mathbf{S}_{\varphi}$ and $\mathbf{S}_{\theta}$ are not self-inverse. The losslessness and reciprocity properties of $\mathbf{S}_{\varphi}$ and $\mathbf{S}_{\theta}$, instead, can be derived similarly to what done in Section III-B, as shown in the following. 
1) Losslessness: Since the reference $N$-port connection network is lossless, condition (23) holds. As far as BWDF based on wave definition (50) are concerned, by replacing (51), (52) and eq. $\mathbf{b}=\mathbf{S}_{\varphi} \mathbf{a}$ into [23, we obtain

$$
W=\frac{1}{\mu^{2}} \mathbf{a}^{T} \mathbf{L}_{\varphi} \mathbf{a}=0
$$

where

$$
\mathbf{L}_{\varphi}=\left(\mathbf{R}_{\alpha}+\mathbf{S}_{\varphi}^{T} \mathbf{R}_{\beta}\right) \Phi^{2}\left(\mathbf{R}_{\alpha}+\mathbf{R}_{\beta}\right)^{-2}\left(\mathbf{I}-\mathbf{S}_{\varphi}\right) .
$$

From that follows the following losslessness property

$$
\mathbf{L}_{\varphi}=\mathbf{0}
$$

A similar result is derived for BWDFs based on the wave definition (75). By replacing (76), (77) and eq. $\mathbf{b}=\mathbf{S}_{\theta} \mathbf{a}$ into (23), we obtain

$$
W=\frac{1}{\mu^{2}} \mathbf{a}^{T} \mathbf{L}_{\theta} \mathbf{a}=0
$$

where

$$
\mathbf{L}_{\theta}=\left(\mathbf{I}+\mathbf{S}_{\theta}^{T}\right) \boldsymbol{\Theta}^{2}\left(\mathbf{R}_{\alpha}^{-1}+\mathbf{R}_{\beta}^{-1}\right)^{2}\left(\mathbf{R}_{\alpha}^{-1}-\mathbf{R}_{\beta}^{-1} \mathbf{S}_{\theta}\right)
$$

from which we deduce the following losslessness property

$$
\mathbf{L}_{\theta}=\mathbf{0} \text {. }
$$

2) Reciprocity: As far as BWDFs based on wave definition (50) are concerned, by applying definitions (51) and (52) and by replacing eq. $\tilde{\mathbf{b}}=\mathbf{S}_{\varphi} \tilde{\mathbf{a}}$ and eq. $\hat{\mathbf{b}}=\mathbf{S}_{\varphi} \hat{\mathbf{a}}$ into the reciprocity condition $(3)$, we obtain

$$
\tilde{\mathbf{a}}^{T} \mathbf{L}_{\varphi} \hat{\mathbf{a}}=\hat{\mathbf{a}}^{T} \mathbf{L}_{\varphi} \tilde{\mathbf{a}}
$$

where $\mathbf{L}_{\varphi}$ is defined in (83), while $\tilde{\mathbf{a}}$ and $\hat{\mathbf{a}}$ are the incident waves from two distinct signal distributions as defined in 30 and (31). Consequently, the reciprocity property turns out to coincide with the losslessness property (84), i.e. $\mathbf{L}_{\varphi}=\mathbf{0}$.

Similarly, as far as BWDFs based on wave definition (75) are concerned, by applying definitions (76) and (77) and by replacing eq. $\tilde{\mathbf{b}}=\mathbf{S}_{\theta} \tilde{\mathbf{a}}$ and eq. $\hat{\mathbf{b}}=\mathbf{S}_{\theta} \hat{\mathbf{a}}$ into the reciprocity condition (3), we obtain

$$
\tilde{\mathbf{a}}^{T} \mathbf{L}_{\theta} \hat{\mathbf{a}}=\hat{\mathbf{a}}^{T} \mathbf{L}_{\theta} \tilde{\mathbf{a}}
$$

where $\mathbf{L}_{\theta}$ is defined in 87). Again, the reciprocity property coincides with the losslessness property $(86)$, i.e. $\mathbf{L}_{\theta}=\mathbf{0}$.

3) Power-normalized waves: We now show that, in the special case of power-normalized waves, $\mathbf{S}_{\varphi}$ and $\mathbf{S}_{\theta}$ are symmetric matrices. In fact, according to Section IV-A, in the power-normalized case, the normalization matrix $\boldsymbol{\Phi}$ can be defined as

$$
\boldsymbol{\Phi}=\frac{1}{\sqrt{2}}\left(\mathbf{R}_{\alpha}+\mathbf{R}_{\beta}\right)^{1 / 2} .
$$

By replacing (90), e.g., in (72), we obtain

$$
\mathbf{S}_{\varphi}=\mathbf{I}-\left(\mathbf{R}_{\alpha}+\mathbf{R}_{\beta}\right)^{1 / 2} \boldsymbol{\Lambda}_{\beta}\left(\mathbf{R}_{\alpha}+\mathbf{R}_{\beta}\right)^{1 / 2} .
$$

As $\boldsymbol{\Lambda}_{\beta}$ is a symmetric matrix $\left(\boldsymbol{\Lambda}_{\beta}^{T}=\boldsymbol{\Lambda}_{\beta}\right)$, then so is $\left(\mathbf{R}_{\alpha}+\mathbf{R}_{\beta}\right)^{1 / 2} \boldsymbol{\Lambda}_{\beta}\left(\mathbf{R}_{\alpha}+\mathbf{R}_{\beta}\right)^{1 / 2}$. Also $\mathbf{S}_{\varphi}$ is symmetric $\left(\mathbf{S}_{\varphi}=\mathbf{S}_{\varphi}^{T}\right)$, because it is the difference between two symmetric matrices. Similarly, according to Section IV-B in the power-normalized case, the normalization matrix $\Theta$ can be defined as

$$
\Theta=\frac{1}{\sqrt{2}}\left(\mathbf{R}_{\alpha}^{-1}+\mathbf{R}_{\beta}^{-1}\right)^{1 / 2}
$$

By replacing (92), e.g., in (78), we obtain

$$
\mathbf{S}_{\theta}=\left(\mathbf{R}_{\alpha}^{-1}+\mathbf{R}_{\beta}^{-1}\right)^{1 / 2} \mathbf{\Psi}_{\beta}\left(\mathbf{R}_{\alpha}^{-1}+\mathbf{R}_{\beta}^{-1}\right)^{1 / 2}-\mathbf{I} .
$$

Since $\boldsymbol{\Psi}_{\beta}$ is a symmetric matrix, i.e., $\boldsymbol{\Psi}_{\beta}^{T}=\boldsymbol{\Psi}_{\beta}$, then $\left(\mathbf{R}_{\alpha}^{-1}+\mathbf{R}_{\beta}^{-1}\right)^{1 / 2} \boldsymbol{\Psi}_{\beta}\left(\mathbf{R}_{\alpha}^{-1}+\mathbf{R}_{\beta}^{-1}\right)^{1 / 2}$ is also a symmetric matrix. It follows that $\mathbf{S}_{\theta}$ is symmetric $\left(\mathbf{S}_{\theta}=\mathbf{S}_{\theta}^{T}\right)$, as it is the difference between two symmetric matrices.

\section{Design of Generalized WDF Adaptors AND EXAMPLES OF APPLICATION}

In this Section, adaptors based on the generalized definitions of wave variables presented in Sections III] and IV are designed and employed for the WD implementation of some reference circuits. Designing adaptors requires:

- deriving their scattering matrices, using the closed-form formulas presented in the previous two Sections;

- deriving adaptation conditions that make one or more of their ports reflection-free, i.e., finding the values of the free parameters that set the corresponding diagonal entries of the scattering matrices to zero.

It is important to underline that, when working with WD structures based on the generalized wave definition of Section III. only one of the ports of a WD junction can be made reflection free. But when we are working with the biparametric waves discussed in Section IV] an arbitrary number of ports (even all of them) of a WD junction can be simultaneously adapted. The WD scattering relations of the linear one-ports that are used in the WDF and BWDF implementations of the considered reference circuits are collected in Table [I] The first row of Table II refers to ideal voltage sources that cannot be adapted. Instead, WD scattering relations of adapted real voltage sources, resistors, capacitors and inductors, along with the imposed adaptation conditions, are provided in the rows that follow. In both WDFs and BWDFs, discretization for implementing reactances (capacitors and inductors) is performed applying the bilinear transform [2], [23].

For the sake of brevity, only output signals of WD structures based on power-normalized waves will be shown. The corresponding WD structures based on the other types of waves have also been implemented in MATLAB, but with exactly the same results (up to the limits of numerical precision). The output signals given by the WD implementations of the considered reference circuits will be compared to the corresponding signals obtained using the LTspice simulation software, in order to verify the accuracy of the developed digital structures.

\section{A. Bridged-T and Twin-T Adaptors}

Closed-form expressions for the scattering matrix of a WDF bridged-T adaptor are obtained by replacing matrix $\mathbf{F}$ of eq. (10) into eq. (47) or into eq. (49). As $p=q=3$ (as 
TABLE II

WAVE Digital Scattering Relations of EMPloyed CiRCUit ElEMENTS

\begin{tabular}{c|c|c|c|c}
\hline $\begin{array}{c}\text { element } \\
\text { name }\end{array}$ & $\begin{array}{c}\text { constitutive } \\
\text { equation }\end{array}$ & generalized WDF & BWDF based on wave def. [50 & BWDF based on dual wave def. $\sqrt{75}$ \\
\hline $\begin{array}{c}\text { ideal volt. } \\
\text { source } e\end{array}$ & $v=e$ & $\begin{array}{c}b[n]= \\
2 R^{\rho-1} e[n]-a[n]\end{array}$ & $b[n]=(\mu(\alpha+\beta) /(\varphi \beta)) e[n]-(\alpha / \beta) a[n]$ & $b[n]=(\mu(1 / \alpha+1 / \beta) / \theta) e[n]-a[n]$ \\
\hline $\begin{array}{c}\text { adapted real } \\
\text { volt. source } \\
e\end{array}$ & $\begin{array}{c}v= \\
e+R_{\mathrm{e}} i\end{array}$ & $\begin{array}{c}b[n]=R^{\rho-1} e[n] \\
\text { with } R=R_{\mathrm{e}}\end{array}$ & $b[n]=(\mu / \varphi) e[n]$ with $\alpha=R_{\mathrm{e}}$ & $b[n]=(\mu /(\theta \alpha)) e[n]$ with $\alpha=R_{\mathrm{e}}$ \\
\hline $\begin{array}{c}\text { adapted } \\
\text { resistor } R_{\mathrm{e}}\end{array}$ & $v=R_{\mathrm{e}} i$ & $\begin{array}{c}b[n]=0 \text { with } \\
R=R_{\mathrm{e}}\end{array}$ & $b[n]=0$ with $\alpha=R_{\mathrm{e}}$ & $b[n]=0$ with $\alpha=R_{\mathrm{e}}$ \\
\hline $\begin{array}{c}\text { adapted } \\
\text { capacitor } C\end{array}$ & $\begin{array}{c}i= \\
C \mathrm{~d} v / \mathrm{d} t\end{array}$ & $\begin{array}{c}b[n]=a[n-1] \text { with } \\
R=1 /\left(2 C F_{\mathrm{s}}\right)\end{array}$ & $\begin{array}{c}(2 \alpha a[n-1]+(\beta-\alpha) b[n-1]) /(\alpha+\beta) \\
\text { with } \alpha=1 /\left(2 C F_{\mathrm{s}}\right) \\
b[n]=\end{array}$ & $\begin{array}{c}(2 \beta a[n-1]+(\beta-\alpha) b[n-1]) /(\alpha+\beta) \\
\text { with } \alpha=1 /\left(2 C F_{\mathrm{S}}\right)\end{array}$ \\
\hline $\begin{array}{c}\text { adapted } \\
\text { inductor } L\end{array}$ & $\begin{array}{c}v= \\
L \mathrm{~d} i / \mathrm{d} t\end{array}$ & $\begin{array}{c}b[n]=-a[n-1] \\
\text { with } R=2 L F_{\mathrm{s}}\end{array}$ & $\begin{array}{c}-(2 \alpha a[n-1]+(\beta-\alpha) b[n-1]) /(\alpha+\beta) \\
\text { with } \alpha=2 L F_{\mathrm{s}}\end{array}$ & $\begin{array}{c}-(2 \beta a[n-1]+(\beta-\alpha) b[n-1]) /(\alpha+\beta) \\
\text { with } \alpha=2 L F_{\mathrm{s}}\end{array}$ \\
\hline
\end{tabular}

outlined in Subsubsection II-A3, the computational complexity that is needed to compute $\mathbf{S}$ is the same when eq. (47) or eq. (49) are used. Similarly, closed-form expressions for the scattering matrices of BWDF bridged-T adaptors are obtained by replacing (10) in (72) or in 78).

Once scattering matrices are expressed as functions of the free parameters, adaptation conditions can be derived. Table III lists the six adaptation conditions at the six ports of the bridged-T adaptor, where $\Pi_{i j k}=\beta_{i} \beta_{j} \beta_{k}, \Pi_{j k}=\beta_{j} \beta_{k}$ and $i, j, k \in\{1, \ldots, 6\}$ are port indexes. The numbering of ports follows the convention used in Fig. 5(c) The adaptation conditions are the same for all kinds of waves. Only one of such conditions can be imposed at a time in a WDF adaptor, since $R_{n}=\alpha_{n}=\beta_{n}, \forall n$. Instead, all six adaptation conditions can be valid at the same time in a BWDF adaptor.

As an example of application of the derived bridged-T adaptors, we consider the WD implementations in Fig. 8 of the circuit in Fig. 3(a) In Fig. 8(a) a WDF bridged-T adaptor with one adapted port is employed, while in Fig. 8(b) a BWDF bridged-T adaptor with all adapted ports is used. In both cases all the WD one-ports are adapted, except for the ideal input voltage source. The circuit is a notch audio filter with parameters $C_{4}=C_{5}=27 \mathrm{pF}, R_{\mathrm{f}}=820 \mathrm{k} \Omega, R_{\mathrm{m}}=680 \Omega$ and $R_{\text {out }}=1 \mathrm{M} \Omega$. In order to measure the impulse responses of the derived digital filters, the input voltage source $V_{\text {in }}$ is driven by an unitary impulse and the voltage across the resistor $R_{\text {out }}$ is taken as an output signal. Fig. 9(a) and Fig. 9(d) compare the magnitude and the phase of the unitary impulse responses of the circuit in Fig. 3(a) in the frequency domain, obtained with a WDF, a BWDF and the LTspice software. The sampling frequency used in the WD implementations is $F_{\mathrm{s}}=96 \mathrm{kHz}$. As shown in Fig. 9(a) and Fig. 9(d), the compared phase and magnitude responses are identical up to numerical precision.

Similar considerations are valid for the design of twin-T adaptors. Matrix $\mathbf{F}$ in eq. (11) is plugged in eq. (47) or in eq. (49). Also in the case of twin-T adaptors, the computational complexity needed to compute $\mathbf{S}$ is the same when eq. (47) or eq. (49) are used, as $p=q=4$. Adaptation conditions for twin-T adaptors have been derived, but are not shown here for reasons of space.

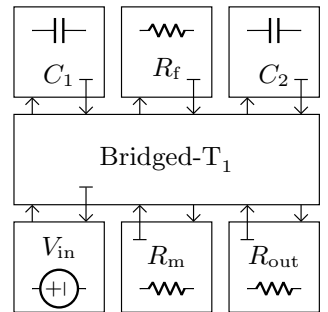

(a)

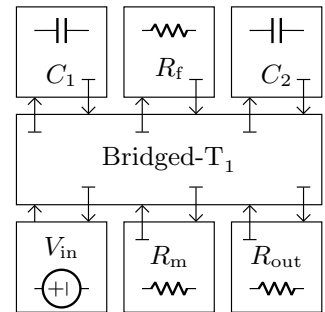

(b)
Fig. 8. WD structures implementing the reference circuit in Fig. 3(a) The WD structure in Fig. 8(a) is based on traditional WDF principles, while the one in Fig. 8(b) is based on BWDF principles.

\section{B. Brune and Darlington-D Adaptors}

Some network synthesis procedures to turn polynomial transfer functions into lumped-element circuits, such as Brune's synthesis [29] or Darlington's synthesis [30], very commonly produce sections which involve absorbed ideal transformers. Two sections of the sort are the Brune and the Darlington-D sections, whose connection networks have been analyzed in Subsection III-B. Corresponding Brune and Darlington-D WDF adaptors are designed as follows. Closedform expressions for their scattering matrices are obtained, considering the $\mathbf{F}$ matrices in eq. (12) and eq. (13) and plugging them in eq. (47) or in eq. (49). In the case of the Brune adaptor, $p=q=2$. Therefore, the same computational cost considerations from the bridged-T adaptor remain valid. Conversely, in the case of the Darlington-D adaptor, $p=3$ and $q=2$; therefore, applying eq. (47) is computationally cheaper than applying eq. (49). Similarly, scattering matrices for BWDFs can be computed, e.g., using eq. (71), eq. (72) or eq. (78). Once expressions for forming scattering matrices are available, adaptation conditions can be derived. Table IV] and Table V list the adaptation conditions of Brune and Darlington$\mathrm{D}$ adaptors, respectively. Also in these two cases, only one of the adaptation conditions can be imposed at a time in WDF adaptors, while, all adaptation conditions can be set at the same time in BWDF adaptors.

In [4], many examples of circuits obtained applying Brune's synthesis or Darlington's synthesis to polynomial transfer functions are shown, along with the corresponding WDF 
TABLE III

ADAPTATION CONDITIONS FOR THE BRIDGED-T ADAPTOR

$\alpha_{1}=\left(\Pi_{425}+\Pi_{426}+\Pi_{423}+\Pi_{453}+\Pi_{463}+\Pi_{256}+\Pi_{253}+\Pi_{563}\right) /\left(\Pi_{45}+\Pi_{46}+\Pi_{43}+\Pi_{25}+\Pi_{26}+\Pi_{56}+\Pi_{23}+\Pi_{63}\right)$
$\alpha_{2}=\left(\Pi_{415}+\Pi_{416}+\Pi_{413}+\Pi_{453}+\Pi_{156}+\Pi_{463}+\Pi_{163}+\Pi_{563}\right) /\left(\Pi_{45}+\Pi_{46}+\Pi_{15}+\Pi_{43}+\Pi_{16}+\Pi_{13}+\Pi_{56}+\Pi_{53}\right)$
$\alpha_{3}=\left(\Pi_{415}+\Pi_{416}+\Pi_{425}+\Pi_{426}+\Pi_{125}+\Pi_{126}+\Pi_{156}+\Pi_{256}\right) /\left(\Pi_{41}+\Pi_{42}+\Pi_{45}+\Pi_{46}+\Pi_{12}+\Pi_{16}+\Pi_{25}+\Pi_{56}\right)$
$\alpha_{4}=\left(\Pi_{125}+\Pi_{126}+\Pi_{156}+\Pi_{123}+\Pi_{256}+\Pi_{163}+\Pi_{253}+\Pi_{563}\right) /\left(\Pi_{15}+\Pi_{16}+\Pi_{25}+\Pi_{13}+\Pi_{26}+\Pi_{23}+\Pi_{53}+\Pi_{63}\right)$
$\alpha_{5}=\left(\Pi_{416}+\Pi_{413}+\Pi_{426}+\Pi_{423}+\Pi_{126}+\Pi_{463}+\Pi_{123}+\Pi_{163}\right) /\left(\Pi_{41}+\Pi_{42}+\Pi_{12}+\Pi_{43}+\Pi_{16}+\Pi_{26}+\Pi_{23}+\Pi_{63}\right)$
$\alpha_{6}=\left(\Pi_{415}+\Pi_{425}+\Pi_{413}+\Pi_{125}+\Pi_{423}+\Pi_{453}+\Pi_{123}+\Pi_{253}\right) /\left(\Pi_{41}+\Pi_{42}+\Pi_{12}+\Pi_{15}+\Pi_{43}+\Pi_{25}+\Pi_{13}+\Pi_{53}\right)$

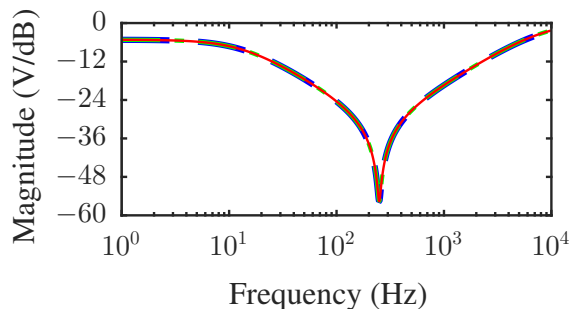

(a)

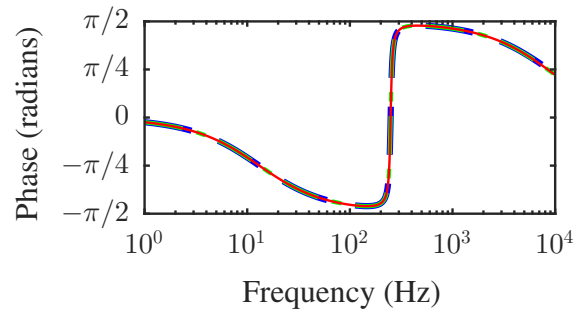

(d)

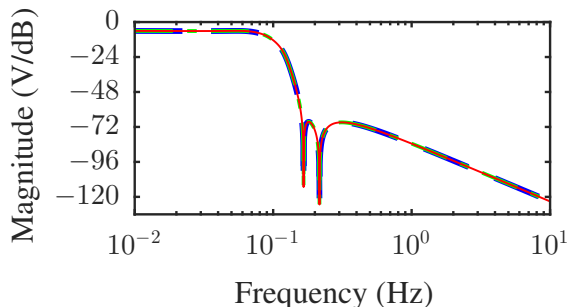

(b)

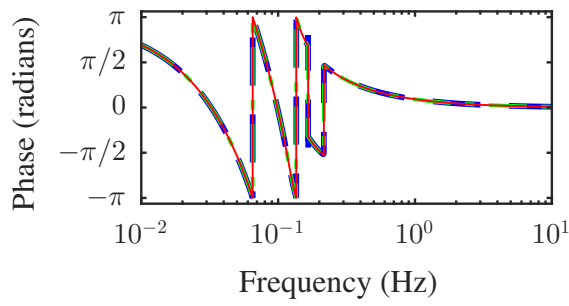

(e)

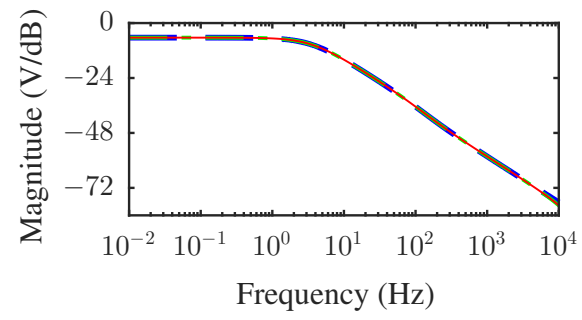

(c)

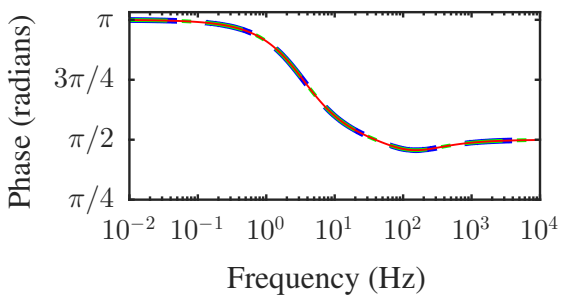

(f)

Fig. 9. Fig. 9(a) and Fig. 9(d) show magnitude and phase of the impulse response of the digital implementations of the reference circuit in Fig. 3(a) The plotted signal is the voltage across resistor $R_{\text {out }}$. Fig. 9(b) and Fig. 9(e) show magnitude and phase of the impulse response of the digital implementations of the reference circuit in Fig. 10 The plotted signal is the voltage across resistor $R_{\text {out }}$. Fig. 9(c) and Fig. 9(f) show magnitude and phase of the impulse response of the digital implementations of the reference circuit containing the real transformer model in Fig. 12 and represented in the WD domain in Fig. 13 The plotted signal is the voltage across resistor $R_{\text {outB. }}$. In each figure, the dashed blue line indicates the result by LTspice, the dashed-dotted green line indicates the result by a traditional WDF based on power-normalized waves and the continuous red line indicates the result by a BWDF based on power-normalized waves; the three plotted lines are always overlapped, indicating that the three different results are matching.

implementations based on voltage waves. One of such circuits, containing two Brune sections, characterized by turns ratios $\eta_{\mathrm{B} 1}=0.14$ and $\eta_{\mathrm{B} 2}=0.16$, respectively, and one DarlingtonD section with turns ratios $\eta_{\mathrm{D} 1}=0.14$ and $\eta_{\mathrm{D} 2}=-1.12$, is reported in Fig. 10. The other circuit parameters are the following: $R_{\text {in }}=1.02 \Omega, R_{\text {out }}=1.02 \Omega, C_{0}=1.61 \mathrm{~F}$, $C_{1}=1.83 \mathrm{~F}, C_{2}=2.26 \mathrm{~F}, C_{3}=5.52 \mathrm{~F}, C_{4}=11.20 \mathrm{~F}$, $L_{0}=0.70 \mathrm{H}, L_{1}=2.12 \mathrm{H}, L_{2}=2.55 \mathrm{H}, L_{3}=3.51 \mathrm{H}$ and $L_{4}=1.60 \mathrm{H}$. Possible WDF and BWDF implementations of the circuit in Fig. 10 are shown in Fig. 11(a) and Fig. 11(b), respectively. The WD inverters $I_{1}$ and $I_{2}$ (equivalent to 2-port series adaptors) make it so that the port polarities match [12], [34], [35]. In order to measure the impulse responses of the derived digital filters, the input voltage source $V_{\text {in }}$ is driven by an unitary impulse and the voltage across the resistor $R_{\text {out }}$ is taken as an output signal. Fig. 9(b) and Fig. 9(e) show how the magnitude and phase responses by LTspice, WDF and BWDF implementations closely match. The sampling frequency used in the WD implementations is $F_{\mathrm{s}}=250 \mathrm{~Hz}$.

\section{Wave Digital Multi-winding Ideal Transformers}

Following the same approach used in the two previous Subsections, WD realizations of multi-winding transformers
TABLE IV

ADAPTATION CONDITIONS FOR THE BRUNE ADAPTOR

$$
\begin{aligned}
& \alpha_{1}=\left(\beta_{3} \beta_{4}\left(\eta_{\mathrm{B}}-1\right)^{2}+\beta_{2} \beta_{3}+\beta_{2} \beta_{4}\right) /\left(\beta_{3} \eta_{\mathrm{B}}^{2}+\beta_{2}+\beta_{4}\right) \\
& \alpha_{2}=\left(\beta_{3} \beta_{4}\left(\eta_{\mathrm{B}}-1\right)^{2}+\beta_{1} \beta_{3} \eta_{\mathrm{B}}^{2}+\beta_{1} \beta_{4}\right) /\left(\beta_{1}+\beta_{3}+\beta_{4}\right) \\
& \alpha_{3}=\left(\beta_{1} \beta_{2}+\beta_{1} \beta_{4}+\beta_{2} \beta_{4}\right) /\left(\beta_{4}\left(\eta_{\mathrm{B}}-1\right)^{2}+\beta_{1} \eta_{\mathrm{B}}^{2}+\beta_{2}\right) \\
& \alpha_{4}=\left(\beta_{1} \beta_{3} \eta_{\mathrm{B}}^{2}+\beta_{1} \beta_{2}+\beta_{2} \beta_{3}\right) /\left(\beta_{3}\left(\eta_{\mathrm{B}}-1\right)^{2}+\beta_{1}+\beta_{2}\right)
\end{aligned}
$$

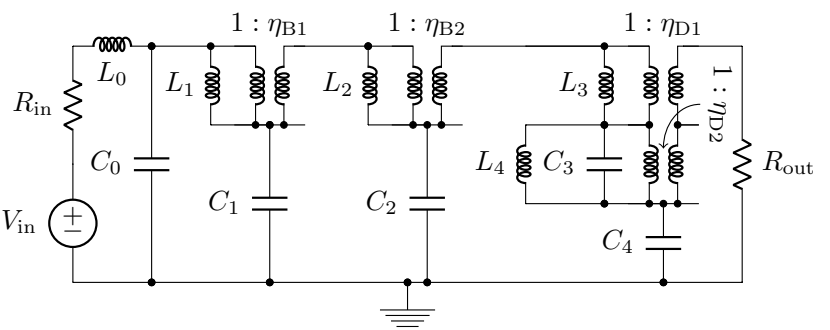

Fig. 10. An example of circuit taken from [4] containing two Brune sections and a Darlington section.

are easily obtained.

We first consider an $N$-port multi-winding transformer based on a series magnetic structure and characterized by the 
TABLE V

ADAPTATION CONDITIONS FOR THE DARLINGTON-D ADAPTOR

$\alpha_{1}=\left(\beta_{2}\left(\beta_{3}+\beta_{4}+\beta_{5}\right)+\beta_{3} \beta_{4}\left(1-\eta_{\mathrm{D} 1}\right)^{2}+\beta_{3} \beta_{5}\left(1-\eta_{\mathrm{D} 2}\right)^{2}+\beta_{4} \beta_{5}\left(\eta_{\mathrm{D} 1}-\eta_{\mathrm{D} 2}\right)^{2}\right) /\left(\beta_{2}+\beta_{3}+\beta_{4} \eta_{\mathrm{D} 1}^{2}+\beta_{5} \eta_{\mathrm{D} 2}^{2}\right)$
$\alpha_{2}=\left(\beta_{1}\left(\beta_{3}+\beta_{4} \eta_{\mathrm{D} 1}^{2}+\beta_{5} \eta_{\mathrm{D} 2}^{2}\right)+\beta_{3} \beta_{4}\left(1-\eta_{\mathrm{D} 1}\right)^{2}+\beta_{3} \beta_{5}\left(1-\eta_{\mathrm{D} 2}\right)^{2}+\beta_{4} \beta_{5}\left(\eta_{\mathrm{D} 1}-\eta_{\mathrm{D} 2}\right)^{2}\right) /\left(\beta_{1}+\beta_{3}+\beta_{4}+\beta_{5}\right)$
$\alpha_{3}=\left(\beta_{1}\left(\beta_{2}+\beta_{4} \eta_{\mathrm{D} 1}^{2}+\beta_{5} \eta_{\mathrm{D} 2}^{2}\right)+\beta_{2}\left(\beta_{4}+\beta_{5}\right)+\beta_{4} \beta_{5}\left(\eta_{\mathrm{D} 1}-\eta_{\mathrm{D} 2}\right)^{2}\right) /\left(\beta_{1}+\beta_{2}+\beta_{4}\left(1-\eta_{\mathrm{D} 1}\right)^{2}+\beta_{5}\left(1-\eta_{\mathrm{D} 2}\right)^{2}\right)$
$\alpha_{4}=\left(\beta_{1}\left(\beta_{2}+\beta_{3}+\beta_{5} \eta_{\mathrm{D} 2}^{2}\right)+\beta_{2}\left(\beta_{3}+\beta_{5}\right)+\beta_{3} \beta_{5}\left(1-\eta_{\mathrm{D} 2}\right)^{2}\right) /\left(\beta_{1} \eta_{\mathrm{D} 1}^{2}+\beta_{2}+\beta_{3}\left(1-\eta_{\mathrm{D} 1}\right)^{2}+\beta_{5}\left(\eta_{\mathrm{D} 1}-\eta_{\mathrm{D} 2}\right)^{2}\right)$
$\alpha_{5}=\left(\beta_{1}\left(\beta_{2}+\beta_{3}+\beta_{4} \eta_{\mathrm{D} 1}^{2}\right)+\beta_{2}\left(\beta_{3}+\beta_{4}\right)+\beta_{3} \beta_{4}\left(1-\eta_{\mathrm{D} 1}\right)^{2}\right) /\left(\beta_{1} \eta_{\mathrm{D} 2}^{2}+\beta_{2}+\beta_{3}\left(1-\eta_{\mathrm{D} 2}\right)^{2}+\beta_{4}\left(\eta_{\mathrm{D} 1}-\eta_{\mathrm{D} 2}\right)^{2}\right)$

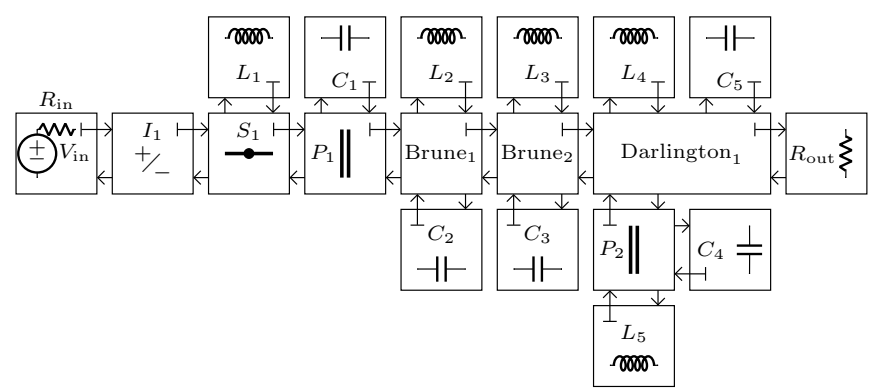

(a)

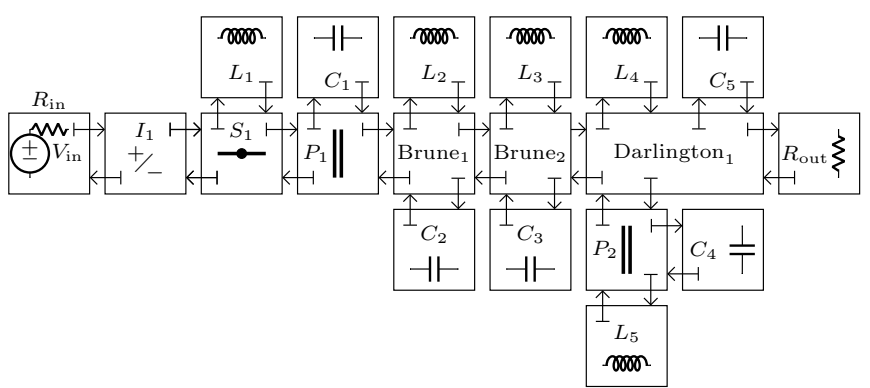

(b)

Fig. 11. Fig. 11(a) and Fig. 11(b) show, respectively, a WDF implementation and a BWDF implementation of the same reference circuit in Fig. 10

F matrix in eq. 16. Since $q<p(q=1$ and $p=N-1)$, using eq. (47) to derive $\mathbf{S}$ is computationally less expensive than using eq. (49), as eq. (47) requires the inversion of a onedimensional linear system, instead of a $(N-1)$-dimensional one. For the same reason, the cheapest formulas for forming the scattering matrices in BWDF implementations are (71) and (78). Once the scattering matrices are formed, adaptation conditions can be derived. A general formula, valid for each type of waves, expressing the adaptation condition at the $n$th port of the $N$-port WD junction is the following

$$
\alpha_{n}=\frac{\gamma_{n}^{2}}{\sum_{k \neq n} \gamma_{k}^{2} / \beta_{k}}
$$

where $\gamma_{k}$ is the $k$ th number of turns as defined in Subsection II-B. It can be verified that, when BWDFs based on the wave definition (75) are used (e.g., (78) is employed), and all $N$ adaptation conditions (94) are set, the entry $s_{\theta}^{i, j}$ at the $i$ th row and $j$ th column of the scattering matrix $\mathbf{S}_{\theta}$ is simply

$$
s_{\theta}^{i, j}= \begin{cases}1, & \text { if } i \neq j \\ 0, & \text { if } i=j .\end{cases}
$$

It is worth noticing that, using BWDFs based on wave definition (75), a scattering matrix $\mathbf{S}_{\theta}$ of a multi-winding transformer with series structure and all adapted ports is equivalent to the scattering matrix of a parallel adaptor with all adapted ports [23].

We then consider the dual case of an $N$-port multi-winding transformer based on a parallel magnetic structure and characterized by the $\mathbf{F}$ matrix in eq. (19). Since $p<q$, being $p=1$ and $q=N-1$, the cheapest formulas for forming the scattering matrices in traditional WDFs and BWDFs are (49), (72) and (79). A general formula, valid for each kind of waves, expressing the adaptation condition at the $n$th port of the $N$-port WD junction is the following

$$
\alpha_{n}=\sum_{k \neq n} \gamma_{n}^{2} \beta_{k} / \gamma_{k}^{2} .
$$

It can be verified that, when BWDFs based on wave definition (50) are used (e.g., (72) is employed), and all $N$ adaptation conditions 96 are set, the entry $s_{\varphi}^{i, j}$ at the $i$ th row and $j$ th column of the scattering matrix $\mathbf{S}_{\varphi}$ is simply given by

$$
s_{\varphi}^{i, j}=\left\{\begin{array}{ll}
-1, & \text { if } i \neq j \\
0, & \text { if } i=j .
\end{array} .\right.
$$

It is worth noticing that, using BWDFs based on wave definition (50), the scattering matrix $\mathbf{S}_{\varphi}$ of a multi-winding transformer with parallel structure and all adapted ports is equivalent to the scattering matrix of a series adaptor with all adapted ports [23].

As an example of application, we provide a WD implementation of the T-model of a real 3-winding transformer in Fig. 12. The T-model is composed of an ideal 3-winding transformer characterized by a series magnetic structure and four inductors. The presented T-model has been used for the emulation of real 3-winding transformers in power electronics systems [36], [37]. However, it could be employed also for modeling multi-winding transformers in audio circuits [38], properly adjusting the values of self and mutual inductances.

We set the following values of self inductances and mutual inductances provided in [36]; $L_{11}=44.35 \mathrm{H}, L_{22}=44.685$ $\mathrm{H}, L_{33}=11.267 \mathrm{H}, M_{12}=42.66 \mathrm{H}, M_{13}=21.301 \mathrm{H}$ and $M_{23}=22.261 \mathrm{H}$. Inductances of T-model are given by $M_{0}=\left(M_{12} M_{13}\right) / M_{23}, L_{10}=L_{11}-M_{0}, L_{1 \mathrm{~A}}=$ $L_{22}-\left(M_{12} M_{23}\right) / M_{13}$ and $L_{\mathrm{lB}}=L_{33}-\left(M_{13} M_{23}\right) / M_{12}$. The ideal 3 -winding transformer is characterized by the turns ratios $\eta_{\mathrm{A}}=M_{23} / M_{13}, \eta_{\mathrm{B}}=M_{23} / M_{12}$, being $\gamma_{1}=1$, $\gamma_{2}=\eta_{\mathrm{A}}$ and $\gamma_{3}=\eta_{\mathrm{B}}$ the numbers of turns. Let us assume 
that a voltage source $V_{\text {in }}$ is connected to the left input port of the T-model. Moreover, two resistors $R_{\text {outA }}=100 \Omega$ and $R_{\text {outB }}=100 \Omega$ are connected to the upper-right and lowerright ports of the T-model, respectively. Possible WDF and BWDF implementations of the resulting circuit are shown in Fig. 13(a) and Fig. 13(b), respectively. The WD inverters $I_{1}$, $I_{2}$ and $I_{3}$ make it so that the port polarities match [12], [34], [35]. In order to measure the impulse responses of the derived digital filters, the input voltage source $V_{\text {in }}$ is driven by an unitary impulse and the voltage across the resistor $R_{\text {outA }}$ is taken as an output signal. Fig. 9(b) and Fig. 9(e) show how the magnitude and phase responses by LTspice, WDF and BWDF implementations are close to each other. The sampling frequency used in the WD implementations is $F_{\mathrm{s}}=48 \mathrm{kHz}$.

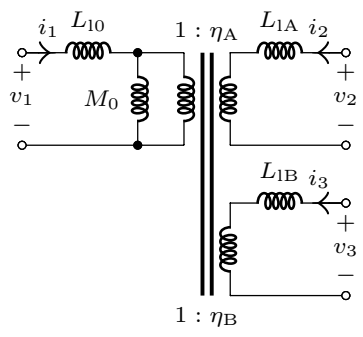

Fig. 12. T-model of a real 3-winding transformer characterized by series structure with turn ratios $\eta_{\mathrm{A}}$ and $\eta_{\mathrm{B}}$.

\section{Discussion, Conclusions, And Future Work}

In this manuscript, we derived closed-form expressions for scattering matrices describing arbitrary reciprocal WD junctions, based on generalized definitions of wave signals. In order to perform a computational cost analysis of such WD junctions, at least two aspects need to be taken into account. The first aspect is the size of the matrix to be inverted in order to form the scattering matrix; the second aspect is the cost of scattering, i.e., computation of the reflected waves given the incident waves, in multiplies. A comparison between the method proposed in this article and the state-of-the-art MNA-based method presented in [7], in terms of these two aspects, is shown in Table VI where $N \geq 2$ is the number of ports of the general reciprocal connection network, $K>1$ is the number of nodes, $q$ is the number of independent port voltages, $p$ is the number of independent port currents and $t_{\eta} \geq 0$ is the total number of turns ratios in case ideal transformers are embedded in the junction. Trivially, the worst possible cost of scattering in multiplies is $N^{2}$. In addition to the general case, all the examples of connection networks discussed in this paper are considered. Table VI shows that the computational efficiency of the proposed method generally surpasses or at least matches that of the method in [7], both when the sizes of matrices to be inverted and the cost of scattering are considered. It is important to say that, according to traditional WDF theory [2], purely series and parallel $N$ ports can always be implemented in the WD domain as interconnections of 3-port series or parallel adaptors, respectively, reducing the computational cost. However, one might prefer to use $N$-port series or parallel junctions in some situations, especially dealing with circuits with multiple nonlinearities [14]. Moreover, studying the computational cost of pure series or parallel junctions allows us to estimate the implementation cost of large reciprocal "series-like" $(p \ll q)$ or "parallel-like" $(q \ll p) N$-ports, for which the WD implementation method proposed in this article is always the best solution. Similar considerations hold true for reciprocal junctions embedding ideal transformers, in which the number of nodes $K$ tends to be comparable to or larger than $N$; also in these cases, the proposed method allows to save computations with respect to the MNA-based method, as confirmed by Table VI

As far as the implementation of arbitrary reciprocal junctions in BWDFs is concerned, a comparison with the state-ofthe-art cannot be performed, as no alternative method exists in the literature. However, their computational cost is similar to that shown in Table VI for $\rho \neq 1 \wedge \rho \neq 0$.

It is also worth mentioning that the expressions of the scattering matrices introduced in this article enable the development of new techniques for efficiently computing waves reflected from the WD junction, without having to actually construct the scattering matrix. A preliminary example of such techniques for regular voltage waves [14] involves the Cholesky factorization and is particularly useful for implementing large circuits. In fact, the possibility of updating WD junctions in an efficient fashion, along with an expanded applicability to all kinds of waves, paves the way to efficiently implementing nonlinear [8], [39]-[44] and/or timevarying networks in which port resistances (free parameters) are frequently changed [14], [15], [45]-[47]. Moreover, the properties of the derived scattering matrices both in traditional WDFs and BWDFs should be further investigated in order to search for extensions of the theorem, proven in [14], granting convergence of the Scattering Iterative Method, applied for solving nonlinear circuits [14], [15].

\section{REFERENCES}

[1] G. O. Martens and K. Meerkötter, "On N-port adaptors for wave digital filters with application to a bridged-tee filter," in Proc. IEEE Int. Symp. Circuits Syst. (ISCAS), Munich, Germany, Apr. 1976, pp. 514-517.

[2] A. Fettweis, "Wave digital filters: Theory and practice," Proc. IEEE, vol. 74, no. 2, pp. 270-327, Feb. 1986.

[3] J. O. Scanlon and A. D. Fagan, "Wave digital C-sections," in Proc. IEEE Int. Symp. Circuits Syst. (ISCAS), Munich, Germany, Apr. 1976, pp. 510-513.

[4] H. H. Lê, "Wave digital adaptors for Brune, Darlington C and D, and twin-T sections,” Ph.D. diss., Univ. Manitoba, Winnipeg, Canada, 1977.

[5] G. O. Martens and H. H. Lê, "Wave digital adapters for reciprocal second-order sections," IEEE Trans. Circuits Syst., vol. 25, no. 12, pp. 1077-1083, Dec. 1978.

[6] M. Jarmasz and G. O. Martens, "Design of canonic wave digital filters using Brune and matched 4-port adaptors," IEEE Trans. Circuits Syst., vol. 34, no. 5, pp. 480-495, May 1987.

[7] K. J. Werner, A. Bernardini, J. O. Smith III, and A. Sarti, "Modeling circuits with arbitrary topologies and active linear multiports using wave digital filters," IEEE Trans. Circuits Syst. I, Reg. Papers, 2018, DOI https://doi.org/10.1109/TCSI.2018.2837912

[8] K. J. Werner, V. Nangia, J. O. Smith III, and J. S. Abel, "Resolving wave digital filters with multiple/multiport nonlinearities," in Proc. 18th Int. Conf. Digital Audio Effects (DAFx-15), Trondheim, Norway, Nov.-Dec. 2015.

[9] K. J. Werner, J. O. Smith III, and J. S. Abel, "Wave digital filters adaptors for arbitrary topologies and multiport linear elements," in Proc. 18th Int. Conf. Digital Audio Effects (DAFx-15), Trondheim, Norway, Nov.-Dec. 2015. 


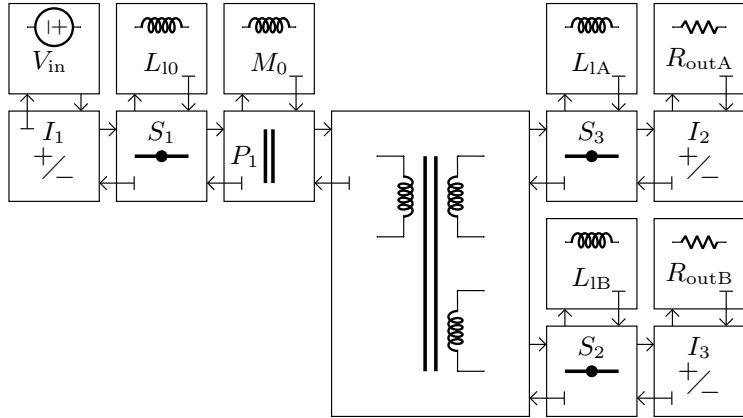

(a)

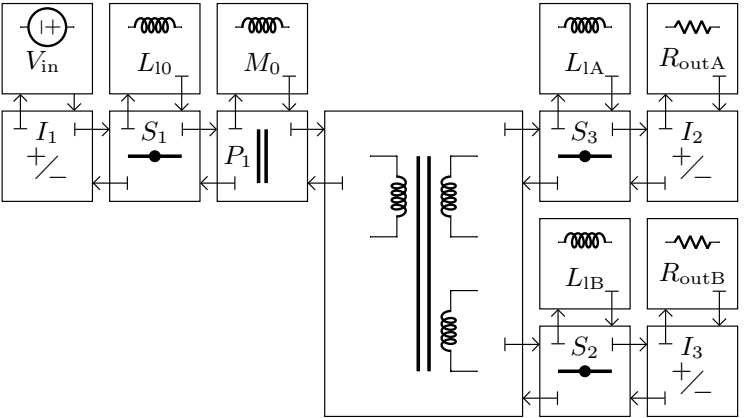

(b)

Fig. 13. Fig. 13(a) shows a WDF implementation of the T-model in Fig. 12 with a connected ideal voltage source and two resistors. Fig. 13(b) shows a BWDF implementation of the same circuit.

TABLE VI

Computational Cost of Reciprocal WD Junctions. Comparison BetweEn Proposed Approach and MNA-BASEd Approach: Minimum Size of Matrix to be inverted and Minimum Cost of SCATtering in Multiplies are Highlighted for EaCh WaVe TyPe.

\begin{tabular}{|c|c|c|c|c|c|c|}
\hline & \multirow{2}{*}{\multicolumn{2}{|c|}{$\frac{\text { Size of Matrix to be Inverted }}{\forall \rho}$}} & \multicolumn{4}{|c|}{ Cost of Scattering in Multiplies } \\
\hline & & & $\rho \neq 1 \wedge$ & $o \neq 0$ & $\rho=1 \vee$ & $=0$ \\
\hline $\begin{array}{l}\text { Connection Network } \\
\text { Name }\end{array}$ & $\begin{array}{l}\text { Proposed } \\
\text { Approach }\end{array}$ & $\begin{array}{l}\text { MNA-based } \\
\text { Approach [7] }\end{array}$ & $\begin{array}{l}\text { Proposed } \\
\text { Approach }\end{array}$ & $\begin{array}{l}\text { MNA-based } \\
\text { Approach [7] }\end{array}$ & $\begin{array}{l}\text { Proposed } \\
\text { Approach }\end{array}$ & $\begin{array}{l}\text { MNA-based } \\
\text { Approach }|7|\end{array}$ \\
\hline General $N$-port & $\begin{array}{l}\min \{q \times q, \\
p \times p\}\end{array}$ & $\begin{array}{l}\left(K-1+t_{\eta}\right) \times \\
\left(K-1+t_{\eta}\right)\end{array}$ & $\begin{array}{l}\min \left\{2 N+2 t_{\eta}+q^{2}\right. \\
\left.2 N+2 t_{\eta}+p^{2}\right\}\end{array}$ & $2 N+(K-1)^{2}$ & $\begin{array}{l}\min \left\{2 N+2 t_{\eta}+q^{2},\right. \\
\left.2 N+2 t_{\eta}+p^{2}\right\}\end{array}$ & $N+(K-1)^{2}$ \\
\hline Series $N$-port & $1 \times 1$ & $(N-1) \times(N-1)$ & $\min \left\{2 N+1, N^{2}\right\}$ & $\begin{array}{l}\min \left\{N^{2},\right. \\
\left.2 N+(N-1)^{2}\right\}\end{array}$ & $N+1$ & $\begin{array}{l}\min \left\{N^{2}\right. \\
\left.N+(N-1)^{2}\right\}\end{array}$ \\
\hline Parallel $N$-port & $1 \times 1$ & $1 \times 1$ & $\min \left\{2 N+1, N^{2}\right\}$ & $\min \left\{2 N+1, N^{2}\right\}$ & $N+1$ & $N+1$ \\
\hline Bridged-T & $3 \times 3$ & $3 \times 3$ & 21 & 21 & 15 & 15 \\
\hline Twin-T & $4 \times 4$ & $4 \times 4$ & 32 & 32 & 24 & 24 \\
\hline Brune Section & $2 \times 2$ & $4 \times 4$ & 14 & 16 & 10 & 13 \\
\hline Darlington Section & $2 \times 2$ & $7 \times 7$ & 16 & 25 & 11 & 25 \\
\hline $\begin{array}{l}N \text {-winding Transformer } \\
\text { with Series Structure }\end{array}$ & $1 \times 1$ & $\begin{array}{l}(3 N-2) \times \\
(3 N-2)\end{array}$ & $\min \left\{4 N-1, N^{2}\right\}$ & $\begin{array}{l}\min \left\{N^{2},\right. \\
\left.2 N+(2 N-1)^{2}\right\}\end{array}$ & $\min \left\{3 N-1, N^{2}\right\}$ & $\begin{array}{l}\min \left\{N^{2},\right. \\
\left.N+(2 N-1)^{2}\right\}\end{array}$ \\
\hline $\begin{array}{l}N \text {-winding Transformer } \\
\text { with Parallel Structure }\end{array}$ & $1 \times 1$ & $\begin{array}{l}(3 N-2) \times \\
(3 N-2)\end{array}$ & $\min \left\{4 N-1, N^{2}\right\}$ & $\begin{array}{l}\min \left\{N^{2}\right. \\
\left.2 N+(2 N-1)^{2}\right\}\end{array}$ & $\min \left\{3 N-1, N^{2}\right\}$ & $\begin{array}{l}\min \left\{N^{2},\right. \\
\left.N+(2 N-1)^{2}\right\}\end{array}$ \\
\hline
\end{tabular}

[10] K. J. Werner, W. R. Dunkel, M. Rest, M. J. Olsen, and J. O. Smith III, "Wave digital filter modeling of circuits with operational amplifiers," in Proc. 24th Eur. Signal Process. Conf. (EUSIPCO), Budapest, Hungary, Aug. 2016, pp. 1033-1037.

[11] M. Verasani, A. Bernardini, and A. Sarti, "Modeling Sallen-Key audio filters in the wave digital domain," in Proc. IEEE Int. Conf. Acoust., Speech Signal Process. (ICASSP), New Orleans, LA, Mar. 2017, pp. 431-435.

[12] K. J. Werner, "Virtual analog modeling of audio circuitry using wave digital filters," Ph.D. diss., Stanford Univ., CA, 2016.

[13] G. Kubin, "Wave digital filters: Voltage, current, or power waves?" in Proc. IEEE Int. Conf. Acoust., Speech Signal Process. (ICASSP), Tampa, Florida, Apr. 1985, pp. 69-72.

[14] A. Bernardini, P. Maffezzoni, L. Daniel, and A. Sarti, "Wave-based analysis of large nonlinear photovoltaic arrays," IEEE Trans. Circuits Syst. I, Reg. Papers, vol. 65, no. 4, pp. 1363-1376, Apr. 2018.

[15] A. Bernardini, K. J. Werner, P. Maffezzoni, and A. Sarti, "Wave digita modeling of the diode-based ring modulator," in Proc. 144nd Conv. Audio Eng. Soc. (AES), Milan, Italy, May 2018, conv. paper \#10015.

[16] A. Bernardini, A. Sarti, P. Maffezzoni, and L. Daniel, "Wave digitalbased variability analysis of electrical mismatch in photovoltaic arrays," in Proc. IEEE Int. Symp. Circuits Syst. (ISCAS), Florence, Italy, May 2018 .

[17] K. Kurokawa, "Power waves and the scattering matrix," IEEE Trans. Microw. Theory Tech., vol. 13, no. 2, pp. 194-202, Mar. 1965.

[18] E. Deprettere and P. Dewilde, "Orthogonal cascade realization of rea multiport digital filters," Int. J. Circuit Theory Appl., vol. 8, no. 3, pp. 245-272, July 1980
[19] P. Vaidyanathan, "A unified approach to orthogonal digital filters and wave digital filters, based on LBR two-pair extraction," IEEE Trans. Circuits Syst., vol. 32, no. 7, pp. 191-236, July 1985.

[20] J. O. Smith III, "Physical modeling using digital waveguides," Comput. Music J. (CMJ), vol. 16, no. 4, pp. 74-91, 1992.

[21] J. O. Smith III, Applications of Digital Signal Processing to Audio and Acoustics. Boston, MA: Springer, 2002, ch. Principles of Digital Waveguide Models of Musical Instruments, pp. 417-466.

[22] S. Bilbao, Wave and Scattering Methods for Numerical Simulation, 1st ed. New York: John Wiley \& Sons, Oct. 2004.

[23] A. Bernardini and A. Sarti, "Biparametric wave digital filters," IEEE Trans. Circuits Syst. I, Reg. Papers, vol. 64, no. 7, pp. 1826-1838, July 2017.

[24] S. Seshu and M. B. Reed, Linear graphs and electrical networks, 1st ed. Reading, MA: Addison-Wesley Publishing Company, 1961.

[25] L. Chua, C. Desoer, and E. Kuh, Linear and Nonlinear Circuits. New York, NY: McGraw-Hill, 1987.

[26] J. L. Bordewijk, "Inter-reciprocity applied to electrical networks," Appl. Sci. Res., vol. 6, no. 1, pp. 1-74, Jan. 1957.

[27] D. Fränken, J. Ochs, and K. Ochs, "Generation of wave digital structures for connection networks containing ideal transformers," in Proc. Int. Symp. Circuits Syst. (ISCAS), vol. 3, May 2003, pp. 240-243.

[28] D. Fränken, J. Ochs, and K. Ochs, "Generation of wave digital structures for networks containing multiport elements," IEEE Trans. Circuits Syst. I, Reg. Papers, vol. 52, no. 3, pp. 586-596, Mar. 2005.

[29] O. Brune, "Synthesis of a finite two-terminal network whose drivingpoint impedance is a prescribed function of frequency," J. Math. Phys., vol. 10, no. 1-4, pp. 191-236, Apr. 1931. 
[30] S. Darlington, "Synthesis of reactance 4-poles which produce prescribed insertion loss characteristics: Including special applications to filter design," J. Math. Phys., vol. 18, no. 1-4, pp. 257-353, 1939.

[31] H. J. Carlin, "Darlington synthesis revisited," IEEE Trans. Circuits Syst. I, Fundam. Theory Appl., vol. 46, no. 1, pp. 14-21, Jan. 1999.

[32] E. C. Cherry, "The duality between interlinked electric and magnetic circuits and the formation of transformer equivalent circuits," Proc. Physical Soc., vol. 62, pp. 101-111, 1949.

[33] K. Meerkötter and D. Fränken, "Digital realization of connection networks by voltage-wave 2-port adapters," Arch. Elek. Übertragung. (AË̈, Int. J. Electron. Commun.), vol. 50, no. 6, pp. 362-367, 1996.

[34] S. D'Angelo and V. Välimäki, "Wave-digital polarity and current inverters and their application to virtual analog audio processing," in Proc. IEEE Int. Conf. Acoust., Speech and Signal Process. (ICASSP), Kyoto, Japan, Mar. 2012, pp. 469-472.

[35] K. J. Werner, W. R. Dunkel, and F. G. Germain, "A computational model of the Hammond organ vibrato/chorus using wave digital filters," in Proc. 19th Int. Conf. Digital Audio Effects (DAFx-16), Brno, Czech Republic, Sept. 2016, pp. 271-278.

[36] J. G. Hayes, N. O'Donovan, and M. G. Egan, "The extended T model of the multiwinding transformer," in Proc. IEEE 35th Annu. Power Electron. Specialists Conf., vol. 3, June 2004, pp. 1812-1817.

[37] X. Margueron and J. P. Keradec, "Design of equivalent circuits and characterization strategy for n-input coupled inductors," IEEE Trans. Ind. Appl., vol. 43, no. 1, pp. 14-22, Jan. 2007.

[38] B. Whitlock, "Audio transformers," in Handbook for Sound Engineers, 4th ed., G. M. Ballou, Ed. Burlington, MA: Focal Press, 2008, ch. 11, pp. 273-307.

[39] A. Bernardini and A. Sarti, "Canonical piecewise-linear representation of curves in the wave digital domain," in Proc. 25th Eur. Signal Proces. Conf. (EUSIPCO), Aug. 2017, pp. 1125-1129.

[40] A. Bernardini, K. J. Werner, A. Sarti, and J. O. Smith III, "Multi-port NonLinearities in wave digital structures," in Proc. IEEE Int. Symp. Signals, Circuits, Syst. (ISSCS), Iași, Romania, 2015.

[41] A. Bernardini, K. J. Werner, A. Sarti, and J. O. Smith III, "Modeling a class of multi-port nonlinearities in wave digital structures," in Proc. 23rd Eur. Signal Process. Conf. (EUSIPCO), Nice, France, Aug. 2015, pp. 669-673.

[42] T. Schwerdtfeger and A. Kummert, "Newton's method for modularitypreserving multidimensional wave digital filters," in Proc. IEEE Int. Work. Multidimensional Syst., Vila Real, Portugal, Sept. 2015.

[43] K. J. Werner, V. Nangia, A. Bernardini, J. O. Smith III, and A. Sarti, "An improved and generalized diode clipper model for wave digital filters," in Proc. 139th Conv. Audio Eng. Soc. (AES), New York, NY, Oct. 2015 conv. paper \#9360.

[44] A. Bernardini, K. J. Werner, A. Sarti, and J. O. Smith III, "Modeling nonlinear wave digital elements using the Lambert function," IEEE Trans. Circuits Syst. I, Reg. Papers, vol. 63, no. 8, pp. 1231-1242, Aug. 2016.

[45] A. Bernardini and A. Sarti, "Dynamic adaptation of instantaneous nonlinear bipoles in wave digital networks," in Proc. 24th Eur. Signal Process. Conf. (EUSIPCO), Budapest, Hungary, Aug. 2016, pp. 10381042.

[46] A. Bernardini, F. Antonacci, and A. Sarti, "Wave digital implementation of robust first-order differential microphone arrays," IEEE Signal Process. Lett., vol. 25, no. 2, pp. 253-257, Feb. 2018.

[47] Ó. Bogason and K. J. Werner, "Modeling time-varying reactances using wave digital filters," in Proc. 21st Int. Conf. Digital Audio Effects (DAFx18), Aveiro, Portugal, Sept. 2018.

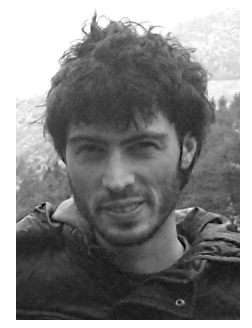

Alberto Bernardini (S'16) received the B.S. degree in computer engineering from the University of Bologna, Italy, in 2012, and the M.S. degree (cum laude) in computer engineering from the Politecnico di Milano, Italy, in 2015, where he is currently pursuing the Ph.D. degree in information engineering with the Dipartimento di Elettronica, Informazione and Bioingegneria. His main research interests are audio signal processing and modeling of nonlinear systems.

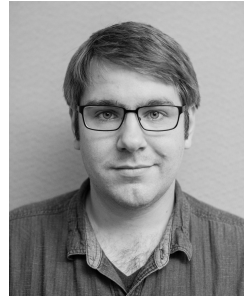

Kurt James Werner (S'14-M'17) received the B.S. degree in general engineering (with a secondary field in acoustics) and the B.Music degree in theory and composition from the University of Illinois at UrbanaChampaign, in 2011, and the Ph.D. degree in computer-based music theory and acoustics from the Center for Computer Research in Music and Acoustics, Stanford University, in 2017. He is currently a Lecturer in audio with the Sonic Arts Research Centre, Queens University Belfast, where he joined the Faculty of Arts, Humanities and Social Sciences and the School of Arts, English and Languages in 2017. His research focuses on theoretical aspects of wave digital filters, virtual analog modeling, computer modeling of circuit-bent instruments, sound synthesis, and the history of music technology. He is a member of the Audio Engineering Society and the International Computer Music Association. He was a recipient of the Best Student Paper Award from the IEEE WASPAA (2015) and (as second author) AES (2017). As a co-author, he received the Best Paper no. 3 (DAFx, 2016) and a Best Paper Honorable Mention (DAFx, 2015).

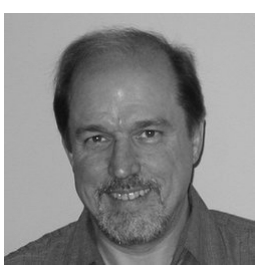

Julius Orion Smith III (M'76) received the B.S.E.E. degree in control, circuits, and communication from Rice University, Houston, TX, USA, in 1975, and the M.S. and Ph.D. degrees in electrical engineering from Stanford University, Stanford, CA, USA, in 1978 and 1983, respectively. His Ph.D. research was devoted to improved methods for digital filter design and system identification applied to music and audio systems. From 1975 to 1977 , he was with the Signal Processing Department, ESL, Sunnyvale, CA, USA, on systems for digital communications. From 1982 to 1986, he was with the Adaptive Systems Department, Systems Control Technology, Palo Alto, CA, USA, where he was involved in the areas of adaptive filtering and spectral estimation. From 1986 to 1991, he was employed at NeXT Computer, Inc., responsible for sound, music, and signal processing software for the NeXT computer workstation. After NeXT, he became an Associate Professor with the Center for Computer Research in Music and Acoustics, Stanford University, teaching courses and pursuing research related to signal processing techniques applied to music and audio systems. Continuing this work, he is currently a Professor of music and (by courtesy) electrical engineering with Stanford University. For more information, see http://ccrma.stanford.edu/jos/.

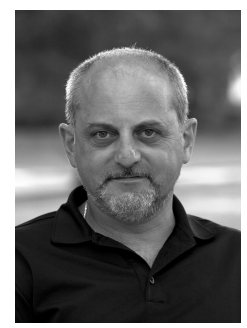

Augusto Sarti (M'04-SM'13) received the M.S. and $\mathrm{Ph} . \mathrm{D}$. degrees in electronics and information engineering from the University of Padua, Italy, in 1988 and 1993, respectively. In 1993, he joined the Faculty of the Politecnico di Milano, Italy, where he is currently a Full Professor. In 2013, he also joined the University of California, Davis. His research interests are in the area of multimedia signal processing, with a particular focus on sound analysis, synthesis and processing, computational acoustics, and music processing. He has co-authored over 250 scientific publications on international journals and congresses and holds numerous patents in the multimedia signal processing area. He coordinates the activities of the Musical Acoustics Lab and the Sound and Music Computing Lab of the Politecnico di Milano. He has been the promoter/coordinator and/or contributor to numerous (20+) European projects. He is an active member of the IEEE Technical Committee on Audio and Acoustics Signal Processing. $\mathrm{He}$ is on the Editorial Board of the IEEE. 